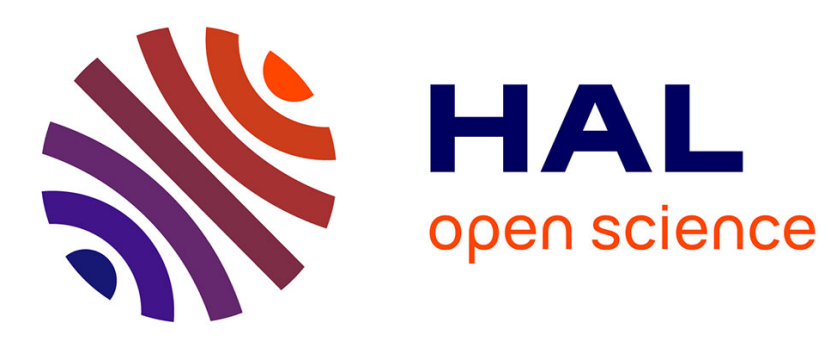

\title{
An Equivariance Theorem with Applications to Renaming
}

Armando Castaneda, Maurice Herlihy, Sergio Rajsbaum

\section{To cite this version:}

Armando Castaneda, Maurice Herlihy, Sergio Rajsbaum. An Equivariance Theorem with Applications to Renaming. [Research Report] PI-1975, 2011, pp.14. inria-00586190

\section{HAL Id: inria-00586190 \\ https://hal.inria.fr/inria-00586190}

Submitted on 15 Apr 2011

HAL is a multi-disciplinary open access archive for the deposit and dissemination of scientific research documents, whether they are published or not. The documents may come from teaching and research institutions in France or abroad, or from public or private research centers.
L'archive ouverte pluridisciplinaire HAL, est destinée au dépôt et à la diffusion de documents scientifiques de niveau recherche, publiés ou non, émanant des établissements d'enseignement et de recherche français ou étrangers, des laboratoires publics ou privés. 


\title{
An Equivariance Theorem with Applications to Renaming
}

\author{
Armando Castañeda $^{*}$, Maurice Herlihy ${ }^{* *}$, Sergio Rajsbaum ${ }^{* * *}$
}

\begin{abstract}
In the renaming problem, each process in a distributed system is issued a unique name from a large name space, and the processes must coordinate with one another to choose unique names from a much smaller name space.

We show that lower bounds on the solvability of renaming in an asynchronous distributed system can be formulated as a purely topological question about the existence of an equivariant chain map from a "topological disk" to a "topological annulus". Proving the non-existence of such a map implies the non-existence of a distributed renaming algorithm in several related models of computation.
\end{abstract}

Key-words: Distributed computing, Renaming, Symmetry breaking, Algebraic topology, Equivariant maps.

\section{Un théorème d'équivariance avec des applications au renommage}

Résumé : Ce rapport présente un théorème d'équivariance avec des applications au renommage.

Mots clés : Informatique distribuée, Renommage, Cassage de symétrie, Topologie algébrique, Cartes équivariantes.

\footnotetext{
* Projet ASAP: équipe commune avec l'INRIA, le CNRS, l'université Rennes 1 et l'INSA de Rennes; armando.castanedar@inria.com.

** Brown University, Computer Science Department, Providence, RI 02912; mph@ cs.brown.edu. Supported by NSF 000830491.

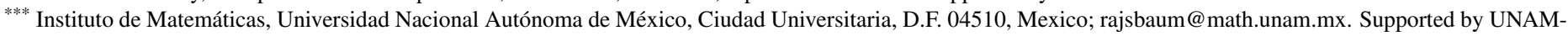
PAPIIT
} 


\section{Introduction}

In the $M$-renaming task, each of $n+1$ processes is issued a unique name taken from a large namespace, and after coordinating with one another, each chooses a unique name taken from a (much smaller) namespace of size M. Processes are asynchronous (there is no bound on their relative speeds), and potentially faulty (any proper subset may halt without warning). Assuming processes communicate through a shared read-write memory, for which values of $M$ can we devise a protocol that ensures that all non-faulty processes choose unique names?

To rule out trivial solutions, we require that any such protocol be anonymous: informally stated, in any execution, the name a process chooses can depend only on the name it was originally issued and how its protocol steps are interleaved with the others.

This problem was first proposed by Attiya et al. [1], who provided a protocol for $M=2 n+1$, and showed that there is no protocol for $M=n+2$. Later, Herlihy and Shavit [9] used chain complexes, a construct borrowed from Algebraic Topology, to show impossibility for $M=2 n$. Unfortunately, this proof, and its later refinements [2, 9, 10], had a flaw: because of a calculation error, the proof did not apply to certain "exceptional" dimensions satisfying a number-theoretic property described below. Castañeda and Rajsbaum [3] provided a new proof based on combinatorial properties of black-and-white simplicial colorings, and were able to show that in these "exceptional" dimensions, and only for them, protocols do exist for $M=2 n$. Nevertheless, this later proof was highly specialized for the weak symmetry breaking task, a task equivalent to $2 n$-renaming, so it was difficult to compare it directly to earlier proofs, either for renaming, or for other distributed problems. In the weak symmetry breaking task [7, 9], each of $n+1$ processes chooses a binary output value, 0 or 1 , such that there is no execution in which the $n+1$ processes choose the same value.

The first contribution of this paper is to formulate the complete renaming proof entirely in the language of Algebraic Topology, using chain complexes and chain maps. While this proof requires more mathematical machinery than the specialized combinatorial arguments used by Castañeda and Rajsbaum, the chain complex formalism is significantly more general. While earlier work has focused on protocols for an asynchronous model where but one process may fail ("wait-free" protocols), the chain complex formalism applies to any model where one can compute the connectivity of the "protocol complexes" associated with that model. This approach has also proved broadly applicable to a range of other problems in Distributed Computing [8, 10]. In this way, we incorporate the renaming task in a broader framework of distributed problems. The second contribution is to point out where the flaw is in previous renaming lower bound proofs $[9,10]$.

As in earlier work $[8,10]$, the existence (or not) of a protocol is equivalent to the existence of a certain kind of chain map between certain chain complexes. Here, we replace the ad-hoc conditions used by prior work [9, 10] to capture the informal notion of anonymity with the well-established mathematical notion of equivariance. Roughly speaking, a map is equivariant if it commutes with actions of a group (in this case, the symmetric group on the set of process IDs). We prove a purely topological theorem characterizing when there exists an equivariant map between the chain complexes of an $n$-simplex and the chain complexes of an annulus. The desired map exists in dimension $n$ if and only if $n$ is not a prime power. These are exactly the dimensions for which renaming is possible for $M=2 n$ [3].

The number-theoretic property used in [3] for stating the renaming solvability result for $M=2 n$, is that the binomial coefficients $\left(\begin{array}{c}n+1 \\ 1\end{array}\right), \ldots,\left(\begin{array}{c}n+1 \\ n\end{array}\right)$ must be relatively prime. In this paper we observe that both properties are equivalent, i.e., $n$ is not a prime power if and only if $\left(\begin{array}{c}n+1 \\ 1\end{array}\right), \ldots,\left(\begin{array}{c}n+1 \\ n\end{array}\right)$ are relatively prime (see for example [4, p. 274]).

\section{Distributed Computing}

We consider a distributed system of $n+1$ processes with distinct IDs taken from $[n]=\{0, \ldots, n\}$. Processes are asynchronous: there is no restriction on their relative speeds. They communicate by writing and reading a shared memory. A task is a distributed problem where each process is issued a private input value, communicates with the other processes, and after taking a bounded number of steps, chooses a private output value and halts. A protocol is a distributed program that solves a task. A protocol is t-resilient if it tolerates crash failures by $t$ of fewer processes, and it is wait-free if it tolerates crash failures by $n$ out of $n+1$ processes.

We model tasks and distributed systems using notions from combinatorial topology [2, 9]. An initial or final state of a process is modeled as a vertex, a pair consisting of a process ID and a value (either input or output). We speak of the vertex as colored with the process ID. A set of $d+1$ mutually compatible initial or final states is modeled as a $d$-dimensional simplex, or $d$-simplex. It is properly colored if the process IDs are distinct. A nonempty subset of a simplex is called a face. An $n$-simplex has $\left(\begin{array}{c}n+1 \\ i+1\end{array}\right)$ faces of dimension $i$.

The complete set of possible initial (or final) states of a distributed task is represented by a set of simplexes, closed under containment, called a simplicial complex, or complex. The dimension of a complex $\mathcal{K}$ is the dimension of a simplex of largest dimension in $\mathcal{K}$. We sometimes use superscripts to indicate dimensions of simplexes and complexes. The set of process IDs associated with a simplex $\sigma^{n}$ is denoted by $\operatorname{ids}\left(\sigma^{n}\right)$, and the set of values by vals $\left(\sigma^{n}\right)$. Sometimes we abuse notation by using $\sigma$ to stand for the complex consisting of $\sigma$ and its faces. The boundary complex bdry $\sigma$ is the complex consisting of proper faces of $\sigma$. For a complex $\mathcal{K}$, its $i$-skeleton, denoted $\operatorname{skel}^{i}(\mathcal{A})$, is the complex containing all simplexes of $\mathcal{A}$ of dimension at most $i$.

A task for $n+1$ processes consists of an input complex $\mathcal{I}^{n}$, and output complex $\mathcal{O}^{n}$ and a map $\triangle$ carrying each input $n$-simplex of $\mathcal{I}^{n}$ to a set of $n$-simplexes of $\mathcal{O}^{n}$. This map associates with each initial state of the system (an input $n$-simplex) the set of legal final states (output $n$-simplexes). It is convenient to extend $\triangle$ to simplexes of lower dimension: $\triangle\left(\sigma^{m}\right)=\cap \triangle\left(\sigma^{n}\right)$, where $\sigma^{n}$ ranges over all $n$-simplexes containing $\sigma^{m}$. This definition has the following operational interpretation: $\triangle\left(\sigma^{m}\right)$ is the set of legal final states in 
executions where only $m+1$ out of $n+1$ processes participate (the rest fail without taking any steps). A protocol solves a task if when the processes run their programs, they start with mutually compatible input values, represented by a simplex $\sigma^{n}$, communicate with one another, and eventually halt with some set of mutually compatible output values, representing a simplex in $\triangle\left(\sigma^{n}\right)$.

Any protocol has an associated protocol complex $\mathcal{P}$, in which each vertex is labeled with a process id and that process's final state (called its view). Each simplex thus corresponds to an equivalence class of executions that "look the same" to the processes at its vertexes. The protocol complex corresponding to executions starting from an input simplex $\sigma^{m}$ is denoted $\mathcal{P}\left(\sigma^{m}\right)$.

A vertex map carries vertexes of one complex to vertexes of another. A simplicial map is a vertex map that preserves simplexes. A simplicial map on properly colored complexes is color-preserving if it associates vertexes of the same color. Let $\mathcal{P}$ be the protocol complex for a protocol. A protocol solves a task $\left\langle\mathcal{I}^{n}, \mathcal{O}^{n}, \triangle\right\rangle$ if and only if there exists a color-preserving simplicial map $\delta: \mathcal{P} \rightarrow \mathcal{O}^{n}$, called a decision map, such that for every $\sigma^{m} \in \mathcal{I}^{n}, \delta\left(\mathcal{P}\left(\sigma^{m}\right)\right) \subset \triangle\left(\sigma^{m}\right)$. We prove our impossibility results by exploiting the topological properties of the protocol complex and the output complex to show that no such map exists.

\section{Algebraic Topology}

Here is a review of some basic notions of algebraic topology (see Munkres [12] or Dieck [5]).

Let $\sigma=\left\{v_{0}, v_{1}, \ldots, v_{q}\right\}$ be a simplex. An orientation of $\sigma$ is a set consisting of a sequence of its vertexes and all even permutations of this sequence. If $n>0$ then these sets fall into two equivalence classes, the sequence $\left\langle v_{0}, v_{1}, \ldots, v_{n}\right\rangle$ and its even permutations, and $\left\langle v_{1}, v_{0}, \ldots, v_{n}\right\rangle$ and its even permutations. Simplexes are oriented in increasing subscript order unless stated otherwise.

A $q$-chain for a complex $\mathcal{K}$ is a formal sum of oriented $q$-simplexes: $\sum_{j=0} \lambda_{j} \sigma_{j}^{q}$, where $\lambda_{j}$ is an integer. Simplexes with zero coefficients are usually omitted, unless they are all zero, when the chain is denoted 0 . We write $1 \cdot \sigma^{q}$ as $\sigma^{q}$ and $-1 \cdot \sigma^{q}$ as $-\sigma^{q}$. For $q>1,-\sigma^{q}$ is identified with $\sigma^{q}$ having the opposite orientation. The $q$-chains of $\mathcal{K}$ form a free Abelian group under component-wise addition, called the $q$-th chain group of $\mathcal{K}$, denoted $\mathcal{C}_{q}(\mathcal{K})$. For dimension -1 , we adjoin the infinite cyclic group $\mathbb{Z}, \mathcal{C}_{-1}(\mathcal{K})=\mathbb{Z}$. We sometimes omit subscripts from chain groups.

A boundary operator $\partial_{q}: \mathcal{C}_{q}(\mathcal{K}) \rightarrow \mathcal{C}_{q-1}(\mathcal{K})$ is a sequence homomorphisms that satisfies $\partial_{q-1} \partial_{q} \alpha=0$. In dimension zero, $\partial_{0}: \mathcal{C}_{0}(\mathcal{K}) \rightarrow \mathcal{C}_{-1}(\mathcal{K})$ must be surjective. For an oriented simplex $\sigma=\left\langle v_{0}, v_{1}, \ldots, v_{q}\right\rangle$, let Face $\sigma$ be the $(q-1)$-face of $\sigma$ after removing vertex $v_{j}$ : Face $\sigma=\left\langle v_{0}, \ldots, \widehat{v_{j}}, \ldots, v_{q}\right\rangle$, where circumflex $\left(^{\frown}\right)$ denotes omission. Let Face ${ }_{i j} \sigma=\left\langle v_{0}, \ldots, \widehat{v_{i}}, \ldots, \widehat{v_{j}}, \ldots, v_{q}\right\rangle$, and so on. For $q>0$, the usual boundary operator $\partial_{q}: \mathcal{C}_{q}(\mathcal{K}) \rightarrow \mathcal{C}_{q-1}(\mathcal{K})$ is defined on simplexes:

$$
\partial_{q} \sigma=\sum_{j=0}^{q}(-1)^{j} \operatorname{Face}_{j}(\sigma)
$$

The boundary operator $\partial_{q}$ extends additively to chains: $\partial_{q}(\alpha+\beta)=\partial_{q} \alpha+\partial_{q} \beta$. For $q=0, \partial_{0}(v)=1$. We sometimes omit subscripts from boundary operators. A $q$-chain $\alpha$ is a boundary if $\alpha=\partial \beta$ for some $(q+1)$-chain $\beta$, and it is a cycle if $\partial \alpha=0$. Since $\partial \partial \alpha=0$, every boundary is a cycle.

The $q$-th homology group of $\mathcal{K}$, denoted $\mathcal{H}_{q}(\mathcal{K})$, is the quotient group

$$
\mathcal{H}_{q}(\mathcal{K})=\operatorname{ker}\left(\partial_{q}\right) / \operatorname{im}\left(\partial_{q+1}\right)
$$

If $\mathcal{H}_{q}(\mathcal{K})=0$ for $q \leq \ell$, we say that $\mathcal{K}$ is $\ell$-acyclic, and if $\mathcal{H}_{q}(\mathcal{K})=0$ for every $q$, we say that $\mathcal{K}$ is acyclic. Two $q$-cycles $\alpha, \alpha^{\prime}$ of $\mathcal{C}_{q}(\mathcal{K})$ are homologous, denoted $\alpha \sim \alpha^{\prime}$, if they belong to the same equivalence class in $\mathcal{H}_{q}(\mathcal{K})$. Equivalently, $\alpha \sim \alpha^{\prime}$ if and only if $\alpha-\alpha^{\prime}$ is a $q$-boundary.

The chain complex of $\mathcal{K}$, denoted $\mathcal{C}(\mathcal{K})$, is the sequence of groups and homomorphisms $\left\{\mathcal{C}_{q}(\mathcal{K}), \partial_{q}\right\}$. Let $\left\{\mathcal{C}_{q}(\mathcal{K}), \partial_{q}\right\}$ and $\left\{\mathcal{C}_{q}(\mathcal{L}), \partial_{q}^{\prime}\right\}$ be chain complexes for $\mathcal{K}$ and $\mathcal{L}$. A chain map $\phi$ is a family of homomorphisms $\phi_{q}: \mathcal{C}_{q}(\mathcal{K}) \rightarrow \mathcal{C}_{q}(\mathcal{L})$, that satisfies $\partial_{q}^{\prime} \circ \phi_{q}=\phi_{q-1} \circ \partial_{q}$. Therefore, $\phi_{q}$ preserves cycles and boundaries. That is, if $\alpha$ is a $q$-cycle $\left(q\right.$-boundary) of $\mathcal{C}_{q}(\mathcal{K}), \phi_{q}(\alpha)$ is a $q$-cycle ( $q$-boundary) of $\mathcal{C}_{q}(\mathcal{L})$. Any simplicial map $\mu: \mathcal{K} \rightarrow \mathcal{L}$ induces a chain map $\mu_{\#}: \mathcal{C}(\mathcal{K}) \rightarrow \mathcal{C}(\mathcal{L})$. (For brevity, $\mu$ denotes both the simplicial map and $\mu_{\#}$.) Similarly, any subdivision induces a chain map.

Let $\mathcal{K}$ and $\mathcal{L}$ be properly-colored complexes. A chain map $\phi: \mathcal{C}(\mathcal{K}) \rightarrow \mathcal{C}(\mathcal{L})$ is color-preserving if each simplex that appears in $a(\sigma)$ is properly colored with the colors of $\sigma$.

Let $\mathcal{G}$ be a finite group and $\mathcal{C}(\mathcal{K})$ be a chain complex. An action of $\mathcal{G}$ on $\mathcal{C}(\mathcal{K})$ is a set $\Phi=\left\{\phi_{g} \mid g \in \mathcal{G}\right\}$ of chain maps $\phi_{g}: \mathcal{C}(\mathcal{K}) \rightarrow$ $\mathcal{C}(\mathcal{K})$ such that for the unit element $e \in \mathcal{G}, \phi_{e}$ is the identity, and for all $g, h \in \mathcal{G}, \phi_{g} \circ \phi_{h}=\phi_{g h}$. For clarity, we write $g(\sigma)$ instead of $\psi_{g}(\sigma)$. The pair $(\mathcal{C}(\mathcal{K}), \Phi)$ is a $\mathcal{G}$-chain complex. When $\Phi$ is understood, we just say that $\mathcal{C}(\mathcal{K})$ is a $\mathcal{G}$-chain complex.

Consider two $\mathcal{G}$-chain complexes $(\mathcal{C}(\mathcal{K}), \Phi)$ and $(\mathcal{C}(\mathcal{L}), \Psi)$. Suppose we have a family of homomorphisms

$$
\mu_{q}: \mathcal{C}_{q}(\mathcal{K}) \rightarrow \mathcal{C}_{p}(\mathcal{L})
$$

where possibly $q \neq p$. We say that $\mu=\left\{\mu_{q}\right\}$ is $G$-equivariant, or just equivariant when $\mathcal{G}$ is understood, if $\mu \circ \phi_{g}=\psi_{g} \circ \mu$ for every $g \in G$. This definition can be extended to a family of homomorphisms as follows. For each dimension each $q$ suppose we have a family of homomorphisms

$$
\mu_{q}^{1}, \ldots, \mu_{q}^{i_{q}}: \mathcal{C}_{q}(\mathcal{K}) \rightarrow \mathcal{C}_{p}(\mathcal{L})
$$


We say that $\mu=\left\{\mu_{q}^{i_{q}}\right\}$ is $G$-equivariant if for every $g \in G$ and for every $\mu^{i} \in \mu, \mu^{j} \circ \phi_{g}=\psi_{g} \circ \mu^{i}$ for some $\mu^{j} \in \mu$.

Let $\mathcal{S}_{n}$ be the symmetric group consisting of all permutations of $[n]$. Henceforth, unless stated otherwise, "equivariant" means " $\mathcal{S}_{n}$-equivariant", where the value of $n$ should be clear from context.

\section{Weak Symmetry-Breaking}

It is convenient to reduce the $2 n$-renaming problem to the following equivalent [7] but simplified form. In the weak symmetry-breaking (WSB) task [7, 9], the processes start with fixed (trivial) inputs, and must choose 0 or 1 such that not all decide 0 and not all decide 1. Just as for renaming, to rule out trivial solutions any protocol for WSB must be anonymous.

We now define the input and output complexes for weak symmetry-breaking. The input complex a combinatorial disk (a single simplex), and the output complex is a combinatorial annulus (a disk with a hole). More precisely, the input complex is a single $n$ simplex $\sigma^{n}$ properly colored with process IDs taken from $[n]$, together with its faces. For brevity, we use $\sigma^{n}$ to refer to this complex. Let $\left\langle P_{0}, P_{1}, \ldots, P_{j}\right\rangle$ denote the oriented face of $\sigma^{n}$ with colors $P_{0}, P_{1}, \ldots, P_{j}$ and with the orientation that contains the sequence $\left\langle P_{0} P_{1} \ldots P_{j}\right\rangle$. Clearly, $\mathcal{C}\left(\sigma^{n}\right)$ is a $\mathcal{S}_{n}$-chain complex: for each $\pi \in \mathcal{S}_{n}, \pi\left(\left\langle P_{0} P_{1} \ldots P_{j}\right\rangle\right)=\left\langle\pi\left(P_{0}\right) \pi\left(P_{1}\right) \ldots \pi\left(P_{j}\right)\right\rangle$.

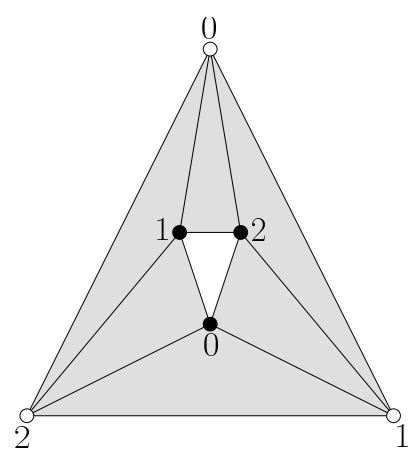

Figure 1: The annulus of dimension 2.

The output complex $\mathcal{A}^{n}$ is defined as follows. Each vertex has the form $\left(P_{i}, b_{i}\right)$, where $P_{i}$ is a process ID and $v_{i}$ is 0 or 1 . A set of vertexes $\left\{\left(P_{0}, v_{0}\right), \ldots,\left(P_{j}, v_{j}\right)\right\}$ defines a simplex of $\mathcal{A}^{n}$ if the $P_{i}$ are distinct, and if $j=n$ then the $b_{i}$ are not all 0 or all 1. This complex is an annulus (Figure 1). Clearly, $\mathcal{C}\left(\mathcal{A}^{n}\right)$ is a $\mathcal{S}_{n}$-chain complex: for each $\pi \in \mathcal{S}_{n}, \pi\left(\left\langle\left(P_{0}, b_{0}\right) \ldots\left(P_{j}, b_{j}\right)\right\rangle\right)=$ $\left\langle\left(\pi\left(P_{0}\right), b_{0}\right) \ldots\left(\pi\left(P_{j}\right), b_{j}\right)\right\rangle$.

\section{An Equivariance Theorem}

As explained below, the existence of a protocol for WSB is tied to the existence of an equivariant chain map from the disk to the annulus. A chain map is non-trivial if it does not send every chain to 0 .

Theorem 5.1. There exists a non-trivial color-preserving equivariant chain map $a: \mathcal{C}\left(\sigma^{n}\right) \rightarrow \mathcal{C}\left(\mathcal{A}^{n}\right)$ if and only if $n$ is not a prime power.

\subsection{Necessity}

This section proves the "only if" direction of Theorem 5.1: if $n$ is a prime power, there is no non-trivial color-preserving equivariant chain map $a: \mathcal{C}\left(\sigma^{n}\right) \rightarrow \mathcal{C}\left(\mathcal{A}^{n}\right)$. We prove that $a$ must map the boundary $\partial \sigma^{n}$ to a cycle of $\mathcal{C}\left(\mathcal{A}^{n}\right)$ that is not a boundary, a contradiction since chain maps preserve cycles and boundaries.

Let $0^{n}$ denote the simplex $\left\{\left\langle P_{0}, 0\right\rangle, \ldots,\left\langle P_{n}, 0\right\rangle\right\}$. Although $0^{n}$ itself is not a simplex of $\mathcal{A}^{n}$, its boundary complex is a subcomplex of $\mathcal{A}^{n}$. Let $\partial 0^{n}$ be the chain $\sum_{i=0}^{n}(-1)^{i}$ Face $_{i} 0^{n}$. This chain is an $(n-1)$-cycle, but not a boundary of $\mathcal{C}\left(\mathcal{A}^{n}\right)$. In fact, $\partial 0^{n}$ is a generator for the homology group of $\mathcal{A}^{n}$, implying that every cycle in $\mathcal{C}\left(\mathcal{A}^{n}\right)$ is homologous to a multiple of $\partial 0^{n}$.

The chain map $z: \mathcal{C}\left(\operatorname{bdry}\left(\sigma^{n}\right)\right) \rightarrow \mathcal{C}\left(\mathcal{A}^{n}\right)$ maps each simplex $\left\langle P_{0} \ldots P_{i}\right\rangle$ of $\mathcal{C}\left(\operatorname{bdry}\left(\sigma^{n}\right)\right)$ to $\left\langle\left\langle P_{0}, 0\right\rangle, \ldots,\left\langle P_{i}, 0\right\rangle\right\rangle$. By construction, $z\left(\partial \sigma^{n}\right)=\partial 0^{n}$.

The proof of the following lemma appears in the appendix.

Lemma 5.2. For each subset $s$ of $[n]$ there is a family of equivariant homomorphisms

$$
\begin{aligned}
d_{q}^{s}: \mathcal{C}_{q}\left(\sigma^{n}\right) & \rightarrow \mathcal{C}_{q+1}\left(\mathcal{A}^{n}\right) \\
f_{p}^{s}: \mathcal{C}_{p}\left(\sigma^{n}\right) & \rightarrow \mathcal{C}_{p}\left(\mathcal{A}^{n}\right)
\end{aligned}
$$


for $-1 \leq q \leq n-2$ and $0 \leq p \leq n-1$, such that for any proper $q$-dimensional face $\sigma$ of $\sigma^{n}$, the chain

$$
a(\sigma)-z(\sigma)-d^{\mathrm{ids}(\sigma)}(\partial \sigma)-\sum_{\sigma^{\prime} \in \operatorname{skel}^{q-2}(\sigma)} f^{\mathrm{ids}\left(\sigma^{\prime}\right)}(\sigma)
$$

is a $q$-cycle.

This construction is similar to the classical notion of a chain homotopy [12], extended here to encompass equivariance.

Using Lemma 5.2 we will show that any color-preserving equivariant chain map $a: \mathcal{C}\left(\sigma^{n}\right) \rightarrow \mathcal{C}\left(\mathcal{A}^{n}\right)$ must map the boundary cycle $\partial \sigma^{n}$ around the "hole" in $\mathcal{A}^{n}$ a non-zero number of times, implying that $a(\partial \sigma)$ is not a boundary. Because $a$ is a chain map, however, it sends boundaries to boundaries, yielding a contradiction.

Theorem 5.3. Let $a: \mathcal{C}\left(\sigma^{n}\right) \rightarrow \mathcal{C}\left(\mathcal{A}^{n}\right)$ be a non-trivial color-preserving equivariant chain map. For some set of integers $k_{0}, \ldots, k_{n-1}$,

$$
a\left(\partial \sigma^{n}\right) \sim\left(1+\sum_{q=0}^{n-1} k_{q}\left(\begin{array}{c}
n+1 \\
q+1
\end{array}\right)\right) \partial 0^{n} .
$$

Proof. By Lemma 5.2, $\alpha_{i}=a\left(\right.$ Face $\left._{i} \sigma\right)-z\left(\right.$ Face $\left._{i} \sigma\right)-d^{\text {ids }\left(\text { Face }_{i} \sigma\right)}\left(\partial\right.$ Face $\left._{i} \sigma\right)-\sum_{\sigma^{\prime} \in \operatorname{skel}^{n-3}\left(\text { Face }_{i} \sigma\right)} f^{\left.\text {ids }^{\prime} \sigma^{\prime}\right)}\left(\right.$ Face $\left._{i} \sigma\right)$ is an $(n-1)-$ cycle. Because $a, z, d$ and $f$ are equivariant, for every $i \in[n], \alpha_{i} \sim(-1)^{i} k_{n-1} \partial 0^{n}$ for some integer $k_{n-1}$. Therefore, $\sum_{i=0}^{q}(-1)^{i} \alpha_{i} \sim$ $k_{n-1}(n+1) \partial 0^{n}$, hence

$$
a\left(\partial \sigma^{n}\right) \sim\left(1+k_{n-1}(n+1)\right) \partial 0^{n}+\gamma+\lambda
$$

where

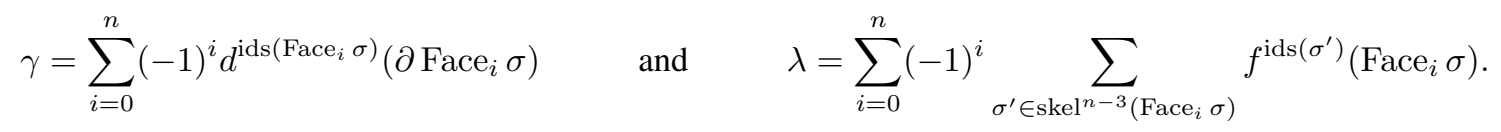

It is a straightforward calculation to show that $\gamma=\sum_{i=0}^{n} \sum_{j+1}^{n} \alpha_{i j}$, where $\alpha_{i j}=(-1)^{i+j}\left(d^{\text {ids }\left(\text { Face }_{j} \sigma\right)}\left(\right.\right.$ Face $\left._{i j} \sigma\right)-d^{\text {ids }\left(\text { Face }_{i} \sigma\right)}\left(\right.$ Face $\left.\left._{i j} \sigma\right)\right)$. Also, it can be proved that $\alpha_{i j}$ is an $(n-1)$-cycle. Moreover, using the fact that $a, z, d$ and $f$ are equivariant, we can show that $\alpha_{i j} \sim k_{n-2} \partial 0^{n}$ for some integer $k_{n-2}$, for every $0 \leq i<j \leq n$. Therefore,

$$
\gamma \sim\left(\begin{array}{c}
n+1 \\
n-1
\end{array}\right) k_{n-2} \partial 0^{n}
$$

We can show that $\lambda=\sum_{\sigma \in \operatorname{skel}^{n-3}\left(\sigma^{n}\right)} \alpha_{\sigma}$, where $\alpha_{\sigma}=\sum_{i \in[n]-\operatorname{ids}(\sigma)}(-1)^{i} f^{\text {ids }(\sigma)}\left(\right.$ Face $\left._{i} \sigma\right)$. Moreover, each $\alpha_{\sigma}$ is an $(n-1)$-cycle. As for $\gamma$, for each $\sigma \in \operatorname{skel}^{n-3}\left(\sigma^{n}\right)$ of dimension $q, \alpha_{\sigma} \sim k_{q} \partial 0^{n}$, for some integer $k_{q}$. Thus,

$$
\lambda \sim \sum_{i=0}^{n-3}\left(\begin{array}{c}
n+1 \\
i+1
\end{array}\right) k_{q} \partial 0^{n}
$$

The theorem follows from Equations (5.1), (5.2) and (5.3).

Theorem 5.3 says that $a\left(\partial \sigma^{n}\right) \sim\left(1+\sum_{q=0}^{n-1} k_{q}\left(\begin{array}{c}n+1 \\ q+1\end{array}\right)\right) \partial 0^{n}$. Because $a$ is a chain map and $\partial \sigma^{n}$ is a boundary, $a\left(\partial \sigma^{n}\right) \sim 0$, implying that $1+\sum_{q=0}^{n-1} k_{q}\left(\begin{array}{c}n+1 \\ q+1\end{array}\right)$ is also 0 . It follows from elementary Number Theory that if $\left(\begin{array}{c}n+1 \\ 1\end{array}\right), \ldots,\left(\begin{array}{c}n+1 \\ n\end{array}\right)$ are not relatively prime, then this equation has no integer solutions. By hypothesis, $n=p^{x}$, for some prime $p$. For $m=1, \ldots, n-1,\left(\begin{array}{c}n \\ m\end{array}\right)=\frac{p^{x} \cdot\left(p^{x}-1\right) \cdots\left(p^{x}-m+1\right)}{1 \cdot 2 \cdots m}=$ $p\left(\frac{p^{x-1} \cdot\left(p^{x}-1\right) \cdots\left(p^{x}-m+1\right)}{1 \cdot 2 \cdots m}\right)$, and since $p$ is prime, we get $\frac{p^{x-1} \cdot\left(p^{x}-1\right) \cdots\left(p^{x}-m+1\right)}{1 \cdot 2 \cdots m}$ is an integer. Thus $p$ is factor of $\left(\begin{array}{c}n \\ m\end{array}\right)$, from which it follows that $\left(\begin{array}{c}n+1 \\ 1\end{array}\right), \ldots,\left(\begin{array}{c}n+1 \\ n\end{array}\right)$ are not relatively prime, a contradiction.

Lemma 5.4. If $n$ is a prime power then there is no non-trivial color-preserving equivariant chain map $a: \mathcal{C}\left(\sigma^{n}\right) \rightarrow \mathcal{C}\left(\mathcal{A}^{n}\right)$.

\subsection{Sufficiency}

In this section we prove the "if" direction of Theorem 5.1: if $n$ is not a prime power, then there is a non-trivial color-preserving equivariant chain map $a: \mathcal{C}\left(\sigma^{n}\right) \rightarrow \mathcal{C}\left(\mathcal{A}^{n}\right)$.

Earlier work [3] presents a construction that takes a simplex $\sigma^{n}$ and a set of integers $\left\{k_{0}, \ldots, k_{n-1}\right\}$ with $k_{0} \in\{0,-1\}$, and produces a subdivision $\chi\left(\sigma^{n}\right)$ with the following two colorings. First, ids is a proper coloring with respect to $[n]$. Second, $b$ is a binary coloring which induces $1+\sum_{i=0}^{n-1} k_{i}\left(\begin{array}{c}n+1 \\ i+1\end{array}\right)$ monochromatic $n$-simplexes. The binary coloring $b$ is symmetric in a sense that for each pair of $m$-faces Face $_{i} \sigma$ and Face $_{j} \sigma$ of $\sigma^{n}$, there is a simplicial bijection $\mu_{i j}: \chi\left(\right.$ Face $\left._{i} \sigma\right) \rightarrow \chi\left(\right.$ Face $\left._{j} \sigma\right)$ such that for every vertex $v \in \chi\left(\operatorname{Face}_{i} \sigma\right), b(v)=b(\mu(v))$ and $\operatorname{rank}(\operatorname{ids}(v))=\operatorname{rank}(\operatorname{ids}(\mu(v)))$, where $\operatorname{rank}: \operatorname{ids}\left(\operatorname{Face}_{i} \sigma\right) \rightarrow \operatorname{ids}\left(\operatorname{Face}_{j} \sigma\right)$ is the rank function such that if $a<b$ in ids $\left(\operatorname{Face}_{i} \sigma\right)$, then $\operatorname{rank}(a)<\operatorname{rank}(b)$. 


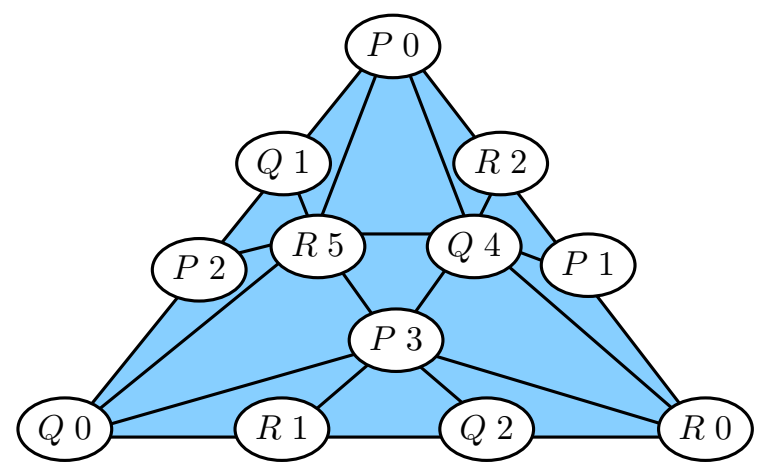

Figure 2: Symmetric input subcomplex for renaming

By a standard construction, subdivisions induce chain maps. In particular, $\chi\left(\sigma^{n}\right)$ induces a chain map $\mu_{1}: \mathcal{C}\left(\sigma^{n}\right) \rightarrow \mathcal{C}\left(\chi\left(\sigma^{n}\right)\right)$. The colorings $i d$ and $b$ define a simplicial map $\chi\left(\sigma^{n}\right) \rightarrow \mathcal{A}^{n}$ only if $b$ defines no monochromatic $n$-simplexes in $\chi\left(\sigma^{n}\right)$. Specifically, if $1+\sum_{i=0}^{n-1} k_{i}\left(\begin{array}{c}n+1 \\ i+1\end{array}\right)=0$. It follows from elementary Number Theory that if $\left(\begin{array}{c}n+1 \\ 1\end{array}\right), \ldots,\left(\begin{array}{c}n+1 \\ n\end{array}\right)$ are relatively prime, then the equation $\left(\begin{array}{c}n+1 \\ 1\end{array}\right) k_{0}+\left(\begin{array}{c}n+1 \\ 2\end{array}\right) k_{1}+\ldots+\left(\begin{array}{c}n+1 \\ n\end{array}\right) k_{n-1}=1$ has an integer solution, thus the simplicial map induced by $i d$ and $b$ induces a chain map $\mu_{2}: \mathcal{C}\left(\chi\left(\sigma^{n}\right)\right) \rightarrow \mathcal{C}\left(\mathcal{A}^{n}\right)$. It is known that if $n$ is not a prime power, then $\left(\begin{array}{c}n+1 \\ 1\end{array}\right), \ldots,\left(\begin{array}{c}n+1 \\ n\end{array}\right)$ are relatively prime (see for example $[4, \mathrm{p}$. 274]).

Let $a$ be the composition $\mu_{2} \circ \mu_{1}$. Since $\chi\left(\sigma^{n}\right)$ is a chromatic subdivision of $\sigma^{n}, a$ is clearly non-trivial and color-preserving. To show that $a$ is equivariant, one can prove by induction on $q$ that the restriction $\left.a\right|_{\mathcal{C}\left(\operatorname{skel}^{q}\left(\sigma^{n}\right)\right)}, 0 \leq q \leq n$, is equivariant. By symmetry of $b$, the base case $q=0$ is trivial. For the induction hypothesis, assume $\left.a\right|_{\mathcal{C}\left(\operatorname{skel}^{q-1}\left(\sigma^{n}\right)\right)}$ is equivariant. The induction step uses the fact that $a$ is color-preserving for proving that, for each $q$-face $\sigma=\left\langle c_{0} \ldots c_{q}\right\rangle$ of $\sigma^{n}, a(\partial \sigma)$ forces the value $a(\sigma)$ such that $\pi \circ a(\sigma)=a \circ \pi(\sigma)$ for every $\pi \in \mathcal{S}_{n}$, hence $\left.a\right|_{\mathcal{C}\left(\operatorname{skel}^{q}\left(\sigma^{n}\right)\right)}$ is equivariant. The proof appears in the appendix.

Lemma 5.5. If $n$ is not a prime power then there is a non-trivial color-preserving equivariant chain map $a: \mathcal{C}\left(\sigma^{n}\right) \rightarrow \mathcal{C}\left(\mathcal{A}^{n}\right)$.

\section{Applications to Distributed Computing}

Theorem 5.1 is a statement about the existence of equivariant chain maps between two simple topological spaces. In this section, we explain what this theorem says about distributed computing.

A complex is $k$-connected if any continuous map from the boundary of a $(k+1)$-simplex to the complex can be extended to a continuous map of the entire simplex. It is known that if a protocol complex $k$-connected, then it cannot solve $(k+1)$-set agreement $[10$, 9]. 1

Here is how to apply this theorem to tell if there is no wait-free protocol for $2 n$-renaming for $(n+1)$ processes in wait-free read-write memory. This description is only a summary: the complete construction appears elsewhere [10]. Recall that WSB and $2 n$-renaming are equivalent in an asynchronous system made of $n+1$ processes that communicate using a read/write shared memory or messagepassing [7].

The WSB task is given by $\left(\sigma^{n}, \mathcal{A}^{n}, \Delta\right)$, where $\sigma^{n}$ is a properly colored simplex that represents the unique input configuration, $\mathcal{A}^{n}$ is the annulus corresponding to all possible output binary values, and $\Delta\left(\sigma^{n}\right)$ defines all legal assignments. Assume we have a wait-free protocol $\mathcal{P}$ that solves WSB, and let $\mathcal{P}\left(\sigma^{n}\right)$ be the complex generated by all executions of the protocol starting from $\sigma^{n}$. Any such protocol complex is $n$-connected [9].

The anonymity requirement for WSB induces a symmetry on the binary output values of the boundary of $\mathcal{P}\left(\sigma^{n}\right)$. This symmetry allows to construct a an equivariant simplicial map $\phi: \mathcal{P}\left(\sigma^{n}\right) \rightarrow \mathcal{P}\left(\mathcal{A}^{n}\right)$. Prepending the map $\mathcal{C}\left(\sigma^{n}\right) \rightarrow \mathcal{C}\left(\mathcal{P}\left(\sigma^{n}\right)\right)$ induced by a subdivision, this equivariant simplicial map induces equivariant chain maps:

$$
\mathcal{C}\left(\sigma^{n}\right) \longrightarrow \mathcal{C}\left(\mathcal{P}\left(\sigma^{n}\right)\right) \longrightarrow \mathcal{C}\left(\mathcal{A}^{n}\right)
$$

The composition of these maps yields an equivariant chain map $a: \mathcal{C}\left(\sigma^{n}\right) \rightarrow \mathcal{C}\left(\mathcal{A}^{n}\right)$. Theorem 5.1, however, states that this chain map does not exist if $n$ is a prime power.

Corollary 6.1. If $n$ is a prime power, there is no wait-free $2 n$-renaming protocol in the asynchronous read/write memory or messagepassing models.

There is a protocol if $n$ is not a prime power [3], but that claim is not implied by this corollary.

\footnotetext{
${ }^{1}$ In the $(k+1)$-set agreement the processes start with a private input value and each chooses an output value among input values; at most $k+1$ distinct output values are elected.
} 
In the more general case, where $t$ out of $n+1$ processes can fail, the construction is a bit more complicated and the dimensions shrink [6]. The $2 n$-renaming task is given by $(\mathcal{I}, \mathcal{O}, \Delta)$, where $\mathcal{I}$ is the complex defining all possible input name assignments, $\mathcal{O}$ is all possible assignments of output names taken from $0, \ldots, 2 n-1$, and for each $\sigma^{n} \in \mathcal{I}, \Delta\left(\sigma^{n}\right)$ defines all legal name assignments.

Assume we have a $t$-resilient $(n+t)$-renaming protocol. Partition the set of processes into two sets, $n-t$ passive processes, and $t+1$ active processes. If $\mathcal{C}$ is a complex labeled with process IDs, let $\mathcal{C}_{a}$ be the subcomplex labeled with IDs of active processes. Let $\mathcal{P}^{*}$ be the protocol complex for executions in which none of the passive processes fail, so all failures are distributed among the active processes. As illustrated in Figure 2, we can identify a subcomplex of $\mathcal{I}$ isomorphic to a subdivision $\chi\left(\sigma^{n}\right)$ of an $n$-simplex $\sigma^{n}$, where the input names are symmetric along the boundary. Because $\mathcal{P}_{a}^{*}\left(\chi\left(\sigma^{n}\right)\right)$ is $t$-connected [11] and by the anonymity requirement for renaming, we can construct a simplicial map $\phi: \chi^{N}\left(\sigma^{t}\right) \rightarrow \mathcal{P}_{a}^{*}\left(\chi\left(\sigma^{n}\right)\right)$ from a subdivision of a $t$-simplex $\sigma^{t}$ to the subcomplex of the restricted protocol complex labeled with active IDs. The simplicial map $\phi$ is equivariant under $\mathcal{S}_{t+1}$, the symmetry group acting on the active process IDs, as is the simplicial decision map $\delta: \mathcal{P}^{*} \rightarrow \mathcal{O}$. It follows that every passive process takes the same output name in every execution of $\mathcal{P}^{*}$. Without loss of generality, assume these passive names are $2 t, \ldots, n+t-1$, leaving the range $0, \ldots, 2 t-1$ to the active processes. Let $\pi: \mathcal{O}_{a} \rightarrow \mathcal{A}^{t}$ send each remaining name to its parity.

These equivariant simplicial maps form a sequence:

$$
\chi^{N}\left(\sigma^{t}\right) \stackrel{\phi}{\longrightarrow} \mathcal{P}_{a}^{*}\left(\chi\left(\sigma^{n}\right)\right) \stackrel{\delta}{\longrightarrow} \mathcal{O}_{a} \stackrel{\pi}{\longrightarrow} \mathcal{A}^{t},
$$

which induces the following sequence of chain maps:

$$
\mathcal{C}\left(\sigma^{t}\right) \longrightarrow \mathcal{C}\left(\mathcal{P}_{a}^{*}\left(\chi\left(\sigma^{n}\right)\right)\right) \longrightarrow \mathcal{C}\left(\mathcal{O}_{a}\right) \longrightarrow \mathcal{C}\left(\mathcal{A}^{t}\right)
$$

The composition of these maps yields an equivariant chain map $a: \mathcal{C}\left(\sigma^{t}\right) \rightarrow \mathcal{C}\left(\mathcal{A}^{t}\right)$. Theorem 5.1, however, states that this chain map does not exist if $t$ is a prime power.

Corollary 6.2. If $t$ is a prime power, there is no $t$-resilient $(n+t)$-renaming protocol in the asynchronous read-write memory or message-passing models.

The extended version of the BG simulation [6] can be used to transform a wait-free protocol to a $t$-resilient protocol. Gafni [6] proves that if WSB is wait-free solvable on $t+1$ processes, then $(n+t)$-renaming is $2 t$-resilient solvable on $n+1$ processes. $^{2}$ Therefore, if $t$ is not a prime power, then $(n+t)$-renaming is $2 t$-resilient solvable on $n+1$ processes, since WSB is wait-free solvable, provided that $t$ is not a prime power.

\section{Previous Renaming Lower Bound Proofs}

As explained in the Introduction, two algebraic renaming lower bound proof stating that $M$-renaming is not wait-free solvable if $M<2 n+1$, are presented in $[9,10]$. This section explains where the flaw is in these proofs.

In [9] it is proved that a wait-free WSB protocol (called reduced renaming in that paper) implies the existence of a color-preserving $S_{n}$-equivariant chain map $a: \mathcal{C}\left(\sigma^{n}\right) \rightarrow \mathcal{C}\left(\mathcal{A}^{n}\right)$. Then it is proved that $a$ cannot exist for any value of $n$. Therefore, WSB is not wait-free solvable, hence neither $2 n$-renaming.

The proof that $a$ does not exist is based on Lemma 6.12 of that paper, which says that that there are homomorphisms $d_{q}: \mathcal{C}_{q}\left(\sigma^{n}\right) \rightarrow$ $\mathcal{C}_{q+1}\left(\mathcal{A}^{n}\right),-1 \leq q \leq n-2$, such that $d=\left\{d_{q}\right\}$ is $S_{n}$-equivariant and for any proper face $\sigma$ of $\sigma^{n}$, the chain $a(\sigma)-z(\sigma)-d(\partial \sigma)$ is a $\operatorname{dim}(\sigma)$-cycle (see sec. 5.1 for the definition of $z$ ). Essentially, $d$ is an equivariant chain homotopy from the restriction $a \mid \mathcal{C}\left(b d\left(\sigma^{n}\right)\right)$ to $z$. Then, using $d$, the paper shows that $a\left(\partial \sigma^{n}\right) \sim(1+(n+1) k) \partial 0^{n}$, for some integer $k$. Since there is no integer $k$ such that $(1+(n+1) k)$ is zero, $a\left(\partial \sigma^{n}\right)$ is not a boundary, for any value of $n$.

The problem with Lemma 6.12 in [9] is that this equivariant $d$ may not exist. Consider a permutation $\pi \in S_{n}$. A chain map $\pi$ partitions the simplexes of $\mathcal{C}\left(\sigma^{n}\right)$ and $\mathcal{C}\left(\mathcal{A}^{n}\right)$ into orbits: the orbit of a simplex $\sigma$ of $\mathcal{C}\left(\sigma^{n}\right)$ or $\mathcal{C}\left(\mathcal{A}^{n}\right)$ is the set containing the simplexes $\pi^{j}(\sigma)$ for $j \geq 0$, where $\pi^{j}$ denotes the $j$-fold composition of $\pi$. Consider a proper face $\sigma$ of $\sigma^{n}$. We have that $d(\sigma)$ has the form $\sum \lambda_{i} \tau_{i}$. The problem comes when the orbits of $\sigma$ and some $\tau_{i}$ are of distinct size. Consider the value of $j$ such that $\pi^{j}(\sigma)=\sigma$. In this case we must have $\pi^{j}\left(\tau_{i}\right)=\tau_{i}$, since $d$ is equivariant. However, it is not true that the orbits of $\sigma$ and $\tau_{i}$ are of same size of for any $\pi \in S_{n}$, as $\sigma$ and $\tau_{i}$ are simplexes of distinct dimension. This precludes to obtain an equivariant $d$.

The renaming lower bound proof of [9] is based on the one in [10], hence the the proof in [10] essentially has the same flaw. Generally speaking, first it is proved that a wait-free $2 n$-renaming algorithm implies the existence of a $\mathbb{Z}_{n}$-equivariant chain map $b: \mathcal{C}\left(\sigma^{n}\right) \rightarrow \mathcal{C}\left(\sigma^{n}\right)$ such that $b\left(\partial \sigma^{n}\right)=0$, where $\mathbb{Z}_{n}$ is the finite cyclic group consisting of $[n]$ under the addition modulo $n+1$ operation.

Then it is claimed that there is a $\mathbb{Z}_{n}$-equivariant chain homotopy $\mathcal{D}$ from $b$ to the identity chain map $i: \mathcal{C}\left(\sigma^{n}\right) \rightarrow \mathcal{C}\left(\sigma^{n}\right)$. Using $\mathcal{D}$, it is proved that $b\left(\partial \sigma^{n}\right)=(1+(n+1) k) \partial \sigma^{n}$, Lemma 6.1 in [10], hence $b\left(\partial \sigma^{n}\right)$ cannot be zero. As in [9], the problem is that it is not true that always there is such equivariant chain homotopy $\mathcal{D}$.

\footnotetext{
${ }^{2}$ This paper [6] uses definitions of WSB and renaming that are different to the definitions we use here, although it claims, without a proof, that the definitions are equivalent.
} 


\section{Acknowledgments}

We thank Ami Paz for pointing to us reference [4] with the equivalence of $n$ not a prime power and $\left(\begin{array}{c}n+1 \\ 1\end{array}\right), \ldots,\left(\begin{array}{c}n+1 \\ n\end{array}\right)$ relatively prime.

\section{References}

[1] H. Attiya, A. Bar-Noy, D. Dolev, D. Peleg \& R. Reischuck. Renaming in Asynchronous Environment. Journal of the ACM 37(3): 524-548 (1990).

[2] H. Attiya \& S. Rajsbaum, The Combinatorial Structure of Wait-Free Solvable Tasks, SIAM Journal on Computing 31(4), pp. 1286-1313, 2002.

[3] A. Castañeda \& S. Rajsbaum. New Combinatorial Topology Upper and Lower Bounds for Renaming. Proceedings of the 27th Annual ACM Symposium on Principles on Distributed Computing, 295-304 (2008).

[4] L. E. Dickson, History Of The Theory Of Numbers I. Carnegie Institution of Washington (1919).

[5] T. tom Dieck. Transformation Groups. Gruiter Studies in Mathematics (1987).

[6] Eli Gafni. The extended BG-simulation and the characterization of t-resiliency, STOC 2009, 85-92.

[7] E. Gafni, S. Rajsbaum \& M. Herlihy. Subconsensus Tasks: Renaming is Weaker than Set Agreement Proceeding of the 20th International Symposium on Distributed Computing, 329-338, (2006).

[8] M. Herlihy, S. Rajsbaum \& M. Tuttle. Unifying Synchronous and Asynchronous Message-Passing Models. Proceedings of the 17th Annual ACM Symposium on Principles of Distributed Computing, 133-142, (1998).

[9] M. Herlihy \& N. Shavit. The Topological Structure of Asynchronous Computability. Journal of the ACM 46(6): 858-923 (1999).

[10] M. Herlihy \& S. Rajsbaum. Algebraic Spans. Mathematical Structures in Computer Science 10(4): 549-573 (2000).

[11] M. Herlihy \& S. Rajsbaum. The topology of shared-memory adversaries. Proceedings of the 29th Annual ACM Symposium on Principles on Distributed Computing, 105-113 (2010).

[12] J. R. Munkres. Elements of Algebraic Topology. Addison-Wesley 1993. 


\section{A Proofs}

\section{A.1 Proofs of Section 5.1}

For distinct $i_{0}, i_{1}, \ldots, i_{q} \in[n], q \leq n-1$, let $\mathcal{S}_{i_{0} i_{1} \ldots i_{q}}^{q}$ denote the subcomplex of $\mathcal{A}^{n}$ that contains all $q$-simplexes, and all its faces, that are properly colored with $i_{0}, i_{1}, \ldots, i_{q}$. It is not hard to see that $\mathcal{S}_{i_{0} i_{1} \ldots i_{q}}^{q}$ is a sphere of dimension $q$.

Lemma A.1. Let $\mathcal{S}$ be a sphere of dimension $n$. Then every $\ell$-cycle is a boundary, $\ell \leq n-1$.

Lemma A.2 ([9]). Let $S_{i}$ be the cycle obtained by orienting each $(n-1)$-simplex of $\mathcal{S}_{0 \ldots i . . . n}^{n-1}$. Then, every $(n-1)$-cycle of $\mathcal{C}\left(\mathcal{O}^{n}\right)$ is homologous to $k S$ for some integer $k$.

Lemma A.3 ([9]). Let $S_{i}$ be the cycle obtained by orienting the $(n-1)$-simplexes of $\mathcal{S}_{0 \ldots \hat{i}}^{n-\ldots n}$ such that its 0 -monochromatic $(n-1)$ simplex is oriented in increasing ids order. Then, $S_{i} \sim(-1)^{i} \partial 0^{n}$.

In what follows, for $0 \leq i \leq m \leq n$, let $\pi_{i}^{m}$ denote the permutation defined as follows:

$$
\pi_{i}^{m}=\left(\begin{array}{cccccccccc}
0 & \ldots & i-1 & i & \ldots & m-1 & m & m+1 & \ldots & n \\
0 & \ldots & i-1 & i+1 & \ldots & m & i & m+1 & \ldots & n
\end{array}\right)
$$

Lemma 5.2 (Restated) For each subset $s$ of $[n]$ there are families of equivariant homomorphisms

$$
\begin{aligned}
d_{q}^{s} & : \mathcal{C}_{q}\left(\sigma^{n}\right) \rightarrow \mathcal{C}_{q+1}\left(\mathcal{A}^{n}\right) \\
f_{p}^{s} & : \mathcal{C}_{p}\left(\sigma^{n}\right) \rightarrow \mathcal{C}_{p}\left(\mathcal{A}^{n}\right)
\end{aligned}
$$

for $-1 \leq q \leq n-2$ and $0 \leq p \leq n-1$. Moreover, for any proper $q$-dimensional face $\sigma$ of $\sigma^{n}$, the chain

$$
a(\sigma)-z(\sigma)-d^{\operatorname{ids}(\sigma)}(\partial \sigma)-\sum_{\sigma^{\prime} \in \operatorname{skel}^{q-2}(\sigma)} f^{\operatorname{ids}\left(\sigma^{\prime}\right)}(\sigma)
$$

is a $q$-cycle.

Proof. We proceed by induction on the dimension of the faces of $\sigma^{n}$. Unless stated otherwise, $d^{s}=0$ and $f^{s}=0$. For the rest of the proof let $\sigma_{i_{0} i_{1} \ldots i_{j}}$ denote the oriented face $\left\langle i_{0} i_{1} \ldots i_{j}\right\rangle$ of $\sigma^{n}$.

For dimension 0 it is easy to see that, for each 0 -face $\sigma$ of $\sigma^{n}, a(\sigma)-z(\sigma)$ is a 0 -cycle. For dimension 1 , consider the face $\sigma_{0}$ and the set $\{0,1\}$. We have that $a\left(\sigma_{0}\right)-z\left(\sigma_{0}\right)$ is a 0 -cycle. Moreover, since $a$ is color-preserving and by the definition of $z, a\left(\sigma_{0}\right), z\left(\sigma_{0}\right) \in$ $\mathcal{C}\left(\mathcal{S}_{0}^{0}\right)$. By Lemma A.1 and since $\mathcal{S}_{0}^{0} \subset \mathcal{S}_{01}^{1}$, there is a 1-chain $d^{01}\left(\sigma_{0}\right) \in \mathcal{C}\left(\mathcal{S}_{01}^{1}\right)$ such that $\partial d^{01}\left(\sigma_{0}\right)=a\left(\sigma_{0}\right)-z\left(\sigma_{0}\right)$. Now, in order to achieve an equivariant $d$, using $d^{01}\left(\sigma_{0}\right)$ we "symmetrically" define the value of $d$ for each pair of 0 -face $\sigma$ and set $s$ of size 2 such that $\operatorname{ids}(\sigma) \subset s$, namely, $d^{\pi(01)}\left(\pi\left(\sigma_{0}\right)\right)=d^{s}(\sigma)=\pi\left(d^{01}\left(\sigma_{0}\right)\right)$, For example, for the 0-face $\sigma_{1}, d^{\pi_{0}^{1}(01)}\left(\pi_{0}^{1}\left(\sigma_{0}\right)\right)=d^{01}\left(\sigma_{1}\right)=\pi_{0}^{1}\left(d^{01}\left(\sigma_{0}\right)\right)$. Observe that the election of $d^{01}\left(\sigma_{0}\right)$ allows to achieve an equivariant $d$. Thus, we have that $\partial d^{01}\left(\sigma_{0}\right)=a\left(\sigma_{0}\right)-z\left(\sigma_{0}\right)$ and $\partial d^{01}\left(\sigma_{1}\right)=$ $a\left(\sigma_{1}\right)-z\left(\sigma_{1}\right)$, hence

$$
\begin{aligned}
\partial d^{01}\left(\sigma_{1}\right)-\partial d^{01}\left(\sigma_{0}\right) & =a\left(\sigma_{1}\right)-z\left(\sigma_{1}\right)-\left(a\left(\sigma_{0}\right)-z\left(\sigma_{0}\right)\right) \\
\partial d^{01}\left(\partial \sigma_{01}\right) & =a\left(\partial \sigma_{01}\right)-z\left(\partial \sigma_{01}\right) \\
0 & =\partial\left(a\left(\sigma_{01}\right)-z\left(\sigma_{01}\right)-d^{01}\left(\partial \sigma_{01}\right)\right)
\end{aligned}
$$

Thus, $a\left(\sigma_{01}\right)-z\left(\sigma_{01}\right)-d^{01}\left(\partial \sigma_{01}\right)$ is a 1-cycle. This complete the basis of the induction.

Assume the lemma holds for faces of dimension $q-1,0 \leq q \leq n-1$. We also assume the following for each $(q-1)$-dimensional face. For a $p$-face $\sigma=\sigma_{c_{0} \ldots c_{p}}$, let Face $i$ denote the $(p-1)$-face $\sigma_{c_{0} \ldots \widehat{c_{i}} \ldots c_{p}}$ of $\sigma$. Similarly, for $i<j$, let Face $i j=\sigma_{c_{0}}, \ldots, \widehat{c_{i}}, \ldots, \widehat{c_{j}}, \ldots, c_{p}$, and so on. For each $(q-1)$-dimensional face $\sigma=\sigma_{c_{0} \ldots c_{q-1}}$, the following holds.

1) For every $(q-2)$-dimensional face $\sigma^{\prime}$ of $\sigma, d^{\operatorname{ids}(\sigma)}\left(\sigma^{\prime}\right) \in \mathcal{C}\left(\mathcal{S}_{\mathrm{ids}(\sigma)}^{q-1}\right)$, and for each $\ell$-dimensional face $\sigma^{\prime}$ of $\sigma, \ell \leq q-3$, $f^{\operatorname{ids}\left(\sigma^{\prime}\right)}(\sigma) \in \mathcal{C}\left(\mathcal{S}_{\operatorname{ids}(\sigma)}^{q-1}\right)$.

2) For every $(q-2)$-dimensional face $\sigma^{\prime}$ of $\sigma$,

$$
\partial d^{\mathrm{ids}(\sigma)}\left(\sigma^{\prime}\right)=a\left(\sigma^{\prime}\right)-z\left(\sigma^{\prime}\right)-d^{\mathrm{ids}\left(\sigma^{\prime}\right)}\left(\partial \sigma^{\prime}\right)-\sum_{\sigma^{\prime \prime} \in \mathrm{skel}^{q-4}\left(\sigma^{\prime}\right)} f^{\mathrm{ids}\left(\sigma^{\prime \prime}\right)}\left(\sigma^{\prime}\right)
$$

3) For every $(q-3)$-dimensional face $\sigma^{\prime}=$ Face $_{i j} \sigma$ of $\sigma, i<j$,

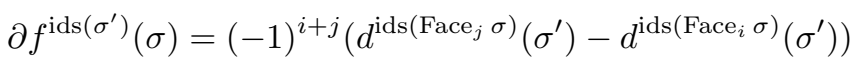


4) For every $k$-dimensional face $\sigma^{\prime}$ of $\sigma, k \leq q-4$,

$$
\partial f^{\operatorname{ids}\left(\sigma^{\prime}\right)}(\sigma)=\sum_{c_{i} \in \operatorname{ids}(\sigma), c_{i} \notin \operatorname{ids}\left(\sigma^{\prime}\right)}(-i)^{i} f^{\operatorname{ids}\left(\sigma^{\prime}\right)}\left(\operatorname{Face}_{i} \sigma\right) .
$$

We prove the lemma holds for faces of dimension $q$. Consider the $q$-simplex $\sigma=\sigma_{0 \ldots q}$. By induction hypothesis,

$$
a\left(\operatorname{Face}_{i} \sigma\right)-z\left(\text { Face }_{i} \sigma\right)-d^{\operatorname{ids}\left(\operatorname{Face}_{i} \sigma\right)}\left(\partial \text { Face }_{i} \sigma\right)-\sum_{\sigma^{\prime} \in \text { skel }^{q-3}\left(\text { Face }_{i} \sigma\right)} f^{{\text {ids }\left(\sigma^{\prime}\right)}^{\prime}\left(\text { Face }_{i} \sigma\right)}
$$

is a $(q-1)$-cycle. Consider the $(q-1)$-dimensional face Face $_{q} \sigma$. By induction hypothesis, for each $(q-2)$-dimensional face $\sigma^{\prime}$ of Face $_{q} \sigma, d^{0 \ldots q-1}\left(\sigma^{\prime}\right) \in \mathcal{C}\left(\mathcal{S}_{0 \ldots q-1}^{q-1}\right)$, and for each $\ell$-dimensional face $\sigma^{\prime}$ of Face $_{q} \sigma, \ell \leq q-3, f^{\text {ids }\left(\sigma^{\prime}\right)}\left(\right.$ Face $\left._{q} \sigma\right) \in \mathcal{C}\left(\mathcal{S}_{0 \ldots q-1}^{q-1}\right)$. Also, $a\left(\right.$ Face $\left._{q} \sigma\right), z\left(\right.$ Face $\left._{q} \sigma\right) \in \mathcal{C}\left(\mathcal{S}_{0 \ldots q-1}^{q-1}\right)$, because $a$ and $z$ are color-preserving. By Lemma A.1 and since $\mathcal{S}_{0 \ldots q-1}^{q-1} \subset \mathcal{S}_{0 \ldots q}^{q}$, there is a $q$-chain $d^{0 \ldots q}\left(\operatorname{Face}_{q} \sigma\right) \in \mathcal{C}\left(\mathcal{S}_{0 \ldots q}^{q}\right)$ such that $\partial d^{0 \ldots q}\left(\right.$ Face $\left._{q} \sigma\right)=a\left(\right.$ Face $\left._{q} \sigma\right)-z\left(\right.$ Face $\left._{q} \sigma\right)-d^{\text {ids }\left(\operatorname{Face}_{q} \sigma\right)}\left(\partial\right.$ Face $\left._{q} \sigma\right)-$ $\sum_{\sigma^{\prime} \in \text { skel }^{q-3}\left(\text { Face }_{q} \sigma\right)} f^{\text {ids }\left(\sigma^{\prime}\right)}\left(\right.$ Face $\left._{q} \sigma\right)$. Using $d^{0 \ldots q}\left(\right.$ Face $\left._{q} \sigma\right)$, we "symmetrically" define the value of $d^{s}\left(\sigma^{\prime}\right)=\pi\left(d^{0 \ldots q}\left(\right.\right.$ Face $\left.\left._{q} \sigma\right)\right)$, where $\operatorname{dim}\left(\sigma^{\prime}\right)=q-1,|s|=q+1, \operatorname{ids}\left(\sigma^{\prime}\right) \subset s, \pi\left(\right.$ Face $\left._{q} \sigma\right)=\sigma^{\prime}$ and $\pi(\{0, \ldots, q\})=s$. Therefore, for each face Face ${ }_{i} \sigma$ of $\sigma$

$$
\partial d^{0 \ldots q}\left(\text { Face }_{i} \sigma\right)=a\left(\text { Face }_{i} \sigma\right)-z\left(\text { Face }_{i} \sigma\right)-d^{\text {ids }\left(\text { Face }_{i} \sigma\right)}\left(\partial \text { Face }_{i} \sigma\right)-\sum_{\sigma^{\prime} \in \text { skel }^{q-3}\left(\text { Face }_{i} \sigma\right)} f^{{\text {ids }\left(\sigma^{\prime}\right)}\left(\text { Face }_{i} \sigma\right)}
$$

and $d^{0 \ldots q}\left(\right.$ Face $\left._{i} \sigma\right) \in \mathcal{C}\left(\mathcal{S}_{0 \ldots q}^{q}\right)$.

Taking the alternating sign sum over all $(q-1)$-faces of $\sigma$, we get

$$
\begin{aligned}
\sum_{i=0}^{q}(-1)^{i} \partial d^{0 \ldots q}\left(\text { Face }_{i} \sigma\right)= & \sum_{i=0}^{q}(-1)^{i}\left\{a\left(\text { Face }_{i} \sigma\right)-z\left(\text { Face }_{i} \sigma\right)\right. \\
& \left.-d^{\text {ids } \left.\text { Face }_{i} \sigma\right)}\left(\partial \text { Face }_{i} \sigma\right)-\sum_{\sigma^{\prime} \in \text { skel }^{q-3}\left(\text { Face }_{i} \sigma\right)} f^{\text {ids }^{\prime}\left(\sigma^{\prime}\right)}\left(\text { Face }_{i} \sigma\right)\right\} \\
\partial d^{0 \ldots q}(\partial \sigma)= & a(\partial \sigma)-z(\partial \sigma)-\gamma-\lambda \\
0= & \partial\left(a(\sigma)-z(\sigma)-d^{0 \ldots q}(\partial \sigma)\right)-\gamma-\lambda
\end{aligned}
$$

where

$$
\gamma=\sum_{i=0}^{q}(-1)^{i} d^{\left.\operatorname{ids} \text { Face }_{i} \sigma\right)}\left(\partial \text { Face }_{i} \sigma\right) \quad \text { and } \quad \lambda=\sum_{i=0}^{q}(-1)^{i} \sum_{\sigma^{\prime} \in \text { skel }^{q-3}\left(\text { Face }_{i} \sigma\right)} f^{\operatorname{ids}\left(\sigma^{\prime}\right)}\left(\text { Face }_{i} \sigma\right) f^{\operatorname{ids}^{\prime}\left(\sigma^{\prime}\right)}\left(\text { Face }_{i} \sigma\right),
$$

We now extend $d$ and $f$ such that

$$
\partial\left(a(\sigma)-z(\sigma)-d^{\mathrm{ids}(\sigma)}(\partial \sigma)\right)-\gamma-\lambda
$$

is a $q$-cycle. First, let us consider $\gamma$. Observe that

$$
\begin{aligned}
& \partial \gamma=\partial \sum_{i=0}^{q}(-1)^{i} d^{\operatorname{ids}\left(\operatorname{Face}_{i} \sigma\right)}\left(\partial \text { Face }_{i} \sigma\right)=\partial \sum_{i=0}^{q}(-1)^{i}\left\{\sum_{j=0}^{i-1}(-1)^{j} d^{\operatorname{ids}\left(\operatorname{Face}_{i} \sigma\right)}\left(\text { Face }_{j i} \sigma\right)\right. \\
& \left.+\sum_{j=i+1}^{q}(-1)^{j-1} d^{\operatorname{ids}\left(\operatorname{Face}_{i} \sigma\right)}\left(\text { Face }_{i j} \sigma\right)\right\} \\
& =\sum_{i=0}^{q} \sum_{j=i+1}^{q}(-1)^{i+j} \partial\left(d^{\left.{\text {ids }\left(\text { Face }_{j} \sigma\right)}_{\text {Face }_{i j} \sigma} \sigma\right)-d^{\mathrm{ids}\left(\text { Face }_{i} \sigma\right)}\left(\text { Face }_{i j} \sigma\right)}\right)
\end{aligned}
$$

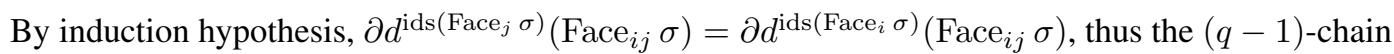
$d^{\text {ids }\left(\text { Face }_{j} \sigma\right)}\left(\right.$ Face $\left._{i j} \sigma\right)-d^{\text {ids }\left(\text { Face }_{i} \sigma\right)}\left(\right.$ Face $\left._{i j} \sigma\right)$ is a cycle. In addition, $d^{\text {ids }\left(\text { Face }_{i} \sigma\right)}\left(\right.$ Face $\left._{i j} \sigma\right) \in \mathcal{C}\left(\mathcal{S}_{\left.\text {ids }^{2-1} \text { Face }_{i} \sigma\right)}\right)$ and $d^{\text {ids }\left(\text { Face }_{j} \sigma\right)}\left(\right.$ Face $\left._{i j} \sigma\right) \in \mathcal{C}\left(\mathcal{S}_{\left.\text {ids }^{-1} \text { Face }_{j} \sigma\right)}\right)$, by induction hypothesis. By Lemma A.1 and since $\mathcal{S}_{\text {ids }^{\left.2-F_{2 a c e} \sigma\right)},} \mathcal{S}_{\left.\text {ids }^{q-1} \text { Face }_{j} \sigma\right)} \subset \mathcal{S}_{0 \ldots q}^{q}$, there exists a $q$-chain $f^{\text {ids }\left(\text { Face }_{i j} \sigma\right)}(\sigma) \in \mathcal{C}\left(\mathcal{S}_{0 \ldots q}^{q}\right)$ such that $\partial f^{\text {ids }\left(\text { Face }_{i j} \sigma\right)}(\sigma)=(-1)^{i+j}\left(d^{\text {ids }\left(\text { Face }_{j} \sigma\right)}\left(\right.\right.$ Face $\left._{i j} \sigma\right)-d^{\text {ids }\left(\text { Face }_{i} \sigma\right)}\left(\right.$ Face $\left.\left._{i j} \sigma\right)\right)$. We use $f^{\left.\text {ids( } \text { Face }_{i j} \sigma\right)}(\sigma)$ to "symmetrically" define the value of $f^{s}\left(\sigma^{\prime}\right)$ for $\operatorname{dim}\left(\sigma^{\prime}\right)=q,|s|=q-1$ and $s \subset \operatorname{ids}\left(\sigma^{\prime}\right)$. So we have

$$
\gamma=\partial \sum_{\sigma^{\prime} \in \operatorname{skel}^{q-2}(\sigma), \operatorname{dim}\left(\sigma^{\prime}\right)=q-2} f^{\operatorname{ids}\left(\sigma^{\prime}\right)}(\sigma)
$$


Consider now $\lambda$. It is not hard to see that

$$
\lambda=\sum_{i=0}^{q}(-1)^{i} \sum_{\sigma^{\prime} \in \text { skel }^{q-3}\left(\text { Face }_{i} \sigma\right)} f^{\operatorname{ids}\left(\sigma^{\prime}\right)}\left(\text { Face }_{i} \sigma\right)=\sum_{\sigma^{\prime} \in \operatorname{skel}^{q-3}(\sigma)} \sum_{i \in[q]-\operatorname{ids}\left(\sigma^{\prime}\right)}(-1)^{i} f^{\operatorname{ids}\left(\sigma^{\prime}\right)}\left(\text { Face }_{i} \sigma\right)
$$

We prove that $\sum_{i \in[q]-\operatorname{ids}\left(\sigma^{\prime}\right)}(-1)^{i} f^{\operatorname{ids}\left(\sigma^{\prime}\right)}\left(\right.$ Face $\left._{i} \sigma\right)$ is a $(q-1)$-cycle and define $f$ for $q$-simplexes. Observe that $\sigma^{\prime}$ is a face of Face ${ }_{i} \sigma$. Fix some $\sigma^{\prime} \in \operatorname{skel}^{q-3}(\sigma)$. We consider two cases, $\operatorname{dim}\left(\sigma^{\prime}\right)=q-3$ and $\operatorname{dim}\left(\sigma^{\prime}\right) \leq q-4$.

Case $\operatorname{dim}\left(\sigma^{\prime}\right)=q-3$. Assume, without loss of generality, $[q]-\operatorname{ids}\left(\sigma^{\prime}\right)=\{a, b, c\}$ with $a<b<c$. We have that

$$
\partial \sum_{i \in[q]-\operatorname{ids}\left(\sigma^{\prime}\right)}(-1)^{i} f^{\operatorname{ids}\left(\sigma^{\prime}\right)}\left(\operatorname{Face}_{i} \sigma\right)=(-1)^{c} \partial f^{\operatorname{ids}\left(\sigma^{\prime}\right)}\left(\operatorname{Face}_{c} \sigma\right)+(-1)^{b} \partial f^{\operatorname{ids}\left(\sigma^{\prime}\right)}\left(\operatorname{Face}_{b} \sigma\right)+(-1)^{a} \partial f^{\operatorname{ids}\left(\sigma^{\prime}\right)}\left(\operatorname{Face}_{a} \sigma\right)
$$

By induction hypothesis,

$$
\begin{aligned}
& \partial f^{\mathrm{ids}\left(\sigma^{\prime}\right)}\left(\text { Face }_{c} \sigma\right)=(-1)^{a+b} f^{\mathrm{ids}\left(\operatorname{Face}_{b c} \sigma\right)}\left(\text { Face }_{a b c} \sigma\right)+(-1)^{a+b-1} f^{\operatorname{ids}\left(\text { Face }_{a c} \sigma\right)}\left(\text { Face }_{a b c} \sigma\right)
\end{aligned}
$$

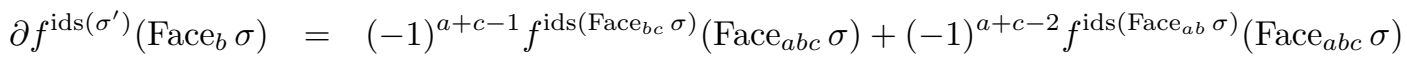

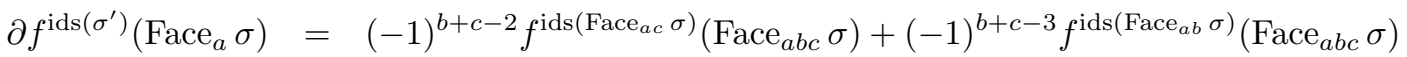

and thus

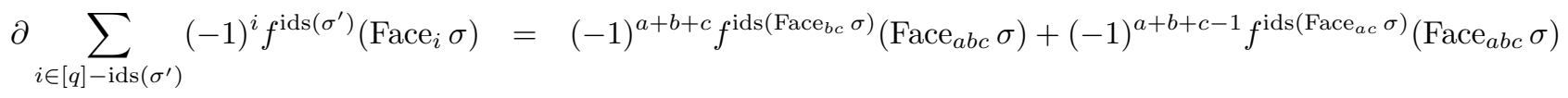

$$
\begin{aligned}
& +(-1)^{a+b+c-1} f^{{\text {ids }\left(\text { Face }_{b c} \sigma\right)}\left(\text { Face }_{a b c} \sigma\right)+(-1)^{a+b+c-2} f^{\text {ids }\left(\text { Face }_{a b} \sigma\right)}\left(\text { Face }_{a b c} \sigma\right)} \\
& +(-1)^{a+b+c-2} f^{\operatorname{ids}\left(\text { Face }_{a c} \sigma\right)}\left(\text { Face }_{a b c} \sigma\right)+(-1)^{a+b+c-3} f^{\text {ids } \left.\text { Face }_{a b} \sigma\right)}\left(\text { Face }_{a b c} \sigma\right) \\
& =0
\end{aligned}
$$

Therefore, $\sum_{i \in[q]-\operatorname{ids}\left(\sigma^{\prime}\right)}(-1)^{i} f^{\mathrm{ids}\left(\sigma^{\prime}\right)}\left(\right.$ Face $\left._{i} \sigma\right)$ is a $(q-1)$-cycle. By induction hypothesis, $f^{\text {ids }\left(\sigma^{\prime}\right)}\left(\operatorname{Face}_{i} \sigma\right) \in \mathcal{C}\left(\mathcal{S}_{\text {ids }^{\prime}\left(\text { Face }_{i} \sigma\right)}\right)$.

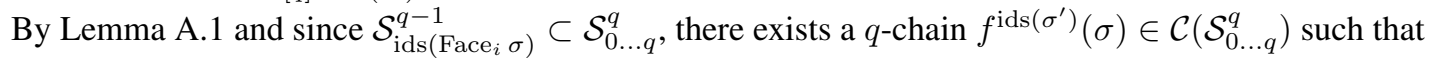
$\partial f^{\operatorname{ids}\left(\sigma^{\prime}\right)}(\sigma)=\sum_{i \in[q]-\operatorname{ids}\left(\sigma^{\prime}\right)}(-1)^{i} f^{\operatorname{ids}\left(\sigma^{\prime}\right)}\left(\right.$ Face $\left._{i} \sigma\right)$. We use $f^{\operatorname{ids}\left(\sigma^{\prime}\right)}(\sigma)$ to "symmetrically" define the value of $f^{s}\left(\sigma^{\prime \prime}\right)$ for $d i m\left(\sigma^{\prime \prime}\right)=$ $q,|s|=q-2$ and $s \subset \operatorname{ids}\left(\sigma^{\prime}\right)$. Therefore,

$$
\sum_{\sigma^{\prime} \in \operatorname{skel}^{q-3}(\sigma), \operatorname{dim}\left(\sigma^{\prime}\right)=q-3} \sum_{i \in[q]-\operatorname{ids}\left(\sigma^{\prime}\right)}(-1)^{i} f^{\operatorname{ids}\left(\sigma^{\prime}\right)}\left(\text { Face }_{i} \sigma\right)=\partial \sum_{\sigma^{\prime} \in \operatorname{skel}^{q-3}(\sigma), \operatorname{dim}\left(\sigma^{\prime}\right)=q-3} f^{\text {ids }\left(\sigma^{\prime}\right)}(\sigma)
$$

Case $\operatorname{dim}\left(\sigma^{\prime}\right) \leq q-4$. By induction hypothesis, for every $i \in[q]-\operatorname{ids}\left(\sigma^{\prime}\right)$,

$$
\partial f^{\operatorname{ids}\left(\sigma^{\prime}\right)}\left(\operatorname{Face}_{i} \sigma\right)=\sum_{j=0, j \notin \operatorname{ids}\left(\sigma^{\prime}\right)}^{i-1}(-1)^{j} f^{\mathrm{ids}\left(\sigma^{\prime}\right)}\left(\operatorname{Face}_{j i} \sigma\right)+\sum_{j=i+1, j \notin \operatorname{ids}\left(\sigma^{\prime}\right)}^{q}(-1)^{j-1} f^{\operatorname{ids}\left(\sigma^{\prime}\right)}\left(\operatorname{Face}_{i j} \sigma\right)
$$

As in the previous case, it can be easily proved that $\sum_{i \in[q]-\operatorname{ids}\left(\sigma^{\prime}\right)}(-1)^{i} f^{\text {ids }\left(\sigma^{\prime}\right)}\left(\right.$ Face $\left._{i} \sigma\right)$ is a $(q-1)$-cycle. Now, by induction

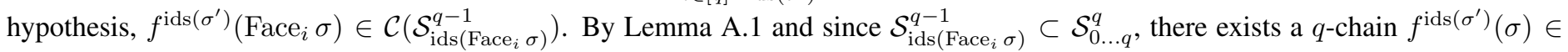
$\mathcal{C}\left(\mathcal{S}_{0 \ldots q}^{q}\right)$ such that $\partial f^{\operatorname{ids}\left(\sigma^{\prime}\right)}(\sigma)=\sum_{i \in[q]-\operatorname{ids}\left(\sigma^{\prime}\right)}(-1)^{i} f^{\text {ids }\left(\sigma^{\prime}\right)}\left(\right.$ Face $\left._{i} \sigma\right)$. We use $f^{\text {ids }\left(\sigma^{\prime}\right)}(\sigma)$ to "symmetrically" define the value of $f^{s}\left(\sigma^{\prime \prime}\right)$ for $\operatorname{dim}\left(\sigma^{\prime \prime}\right)=q,|s| \leq q-3$ and $s \subset \operatorname{ids}\left(\sigma^{\prime \prime}\right)$. Thus, we get

$$
\sum_{\sigma^{\prime} \in \operatorname{skel}^{q-4}(\sigma)} \sum_{i \in[q]-\operatorname{ids}\left(\sigma^{\prime}\right)}(-1)^{i} f^{\operatorname{ids}\left(\sigma^{\prime}\right)}\left(\operatorname{Face}_{i} \sigma\right)=\partial \sum_{\sigma^{\prime} \in \operatorname{skel}^{q-4}(\sigma)} f^{\operatorname{ids}\left(\sigma^{\prime}\right)}(\sigma)
$$

Combining Equations (A.3) and (A.4)

$$
\lambda=\partial \sum_{\sigma^{\prime} \in \text { skel }^{q-3}(\sigma)} f^{\text {ids }\left(\sigma^{\prime}\right)}(\sigma)
$$

Finally, from Equations (A.1), (A.2) and (A.5), we conclude

$$
a(\sigma)-z(\sigma)-d^{\operatorname{ids}(\sigma)}(\partial \sigma)-\sum_{\sigma^{\prime} \in \operatorname{skel}^{q-2}(\sigma)} f^{\operatorname{ids}\left(\sigma^{\prime}\right)}(\sigma)
$$

is a $q$-cycle, hence the lemma holds for faces of dimension $q$. 
Theorem 5.3 (Restated) Let $a: \mathcal{C}\left(\sigma^{n}\right) \rightarrow \mathcal{C}\left(\mathcal{A}^{n}\right)$ be a non-trivial color-preserving equivariant chain map. For some set of integers $k_{0}, \ldots, k_{n-1}$,

$$
a\left(\partial \sigma^{n}\right) \sim\left(1+\sum_{q=0}^{n-1} k_{q}\left(\begin{array}{c}
n+1 \\
q+1
\end{array}\right)\right) \partial 0^{n} .
$$

Proof. Consider the chain map $z: \mathcal{C}\left(\operatorname{bdry}\left(\sigma^{n}\right)\right) \rightarrow \mathcal{C}\left(\mathcal{A}^{n}\right)$ that maps each simplex $\left\langle c_{0} \ldots c_{i}\right\rangle$ of $\mathcal{C}\left(\operatorname{bdry}\left(\sigma^{n}\right)\right)$ to $\left\langle\left(c_{0}, 0\right) \ldots\left(c_{i}, 0\right)\right\rangle$. Observe that $z\left(\sigma^{n}\right)=\partial 0^{n}$. Let $S_{i}$ be the cycle obtained by orienting the $(n-1)$-simplexes of $\mathcal{S}_{0 \ldots \hat{i} \ldots n}^{n-1}$ such that its 0 -monochromatic $(n-1)$-simplex is oriented in increasing ids order. By Lemma 5.2,

$$
\alpha_{i}=a\left(\operatorname{Face}_{i} \sigma\right)-z\left(\operatorname{Face}_{i} \sigma\right)-d^{\operatorname{ids}\left(\operatorname{Face}_{i} \sigma\right)}\left(\partial \text { Face }_{i} \sigma\right)-\sum_{\sigma \in \text { skel }^{n-3}\left(\text { Face }_{i} \sigma\right)} f^{\text {ids }(\sigma)}\left(\text { Face }_{i} \sigma\right)
$$

is an $(n-1)$-cycle. Consider the cycle $\alpha_{n}$. By Lemma A.2, $\alpha_{n} \sim k_{n-1} S_{n}$, for some integer $k_{n-1}$. It is not hard to see that $\pi_{i}^{n}\left(\right.$ Face $\left._{n} \sigma\right)=$ Face $_{i} \sigma$ and $\pi_{i}^{n}\left(S_{n}\right)=S_{i}$. Thus, $\pi_{i}^{n}\left(\alpha_{n}\right)=\alpha_{i}$, because $a, z, d$ and $f$ are equivariant. Therefore, $\pi_{i}^{n}\left(\alpha_{n}\right)=\alpha_{i} \sim$ $k_{n-1} \pi_{i}^{n}\left(S_{n}\right)=k_{n-1} S_{i}$, and by Lemma A.3 we get $\alpha_{i} \sim(-1)^{i} k_{n-1} S_{i}$. Considering the alternating sign sum over all $(n-1)$-faces of $\sigma^{n}$, we get

$$
\begin{aligned}
\sum_{i=0}^{n}(-1)^{i} \quad & \left(a\left(\text { Face }_{i} \sigma\right)-z\left(\text { Face }_{i} \sigma\right)-d^{\text {ids }\left(\text { Face }_{i} \sigma\right)}\left(\partial \text { Face }_{i} \sigma\right)-\sum_{\sigma \in \text { skel }^{n-3}\left(\text { Face }_{i} \sigma\right)} f^{\text {ids } \left.\left.(\sigma)_{\text {(Face }} \sigma\right)\right) \sim}\right. \\
& \sum_{i=0}^{n}(-1)^{i}(-1)^{i} k_{n-1} \partial 0^{n}
\end{aligned}
$$

hence

$$
a\left(\partial \sigma^{n}\right)-z\left(\partial \sigma^{n}\right)-\sum_{i=0}^{n}(-1)^{i} d^{\operatorname{ids}\left(\operatorname{Face}_{i} \sigma\right)}\left(\partial \operatorname{Face}_{i} \sigma\right)-\sum_{i=0}^{n}(-1)^{i} \sum_{\sigma \in \operatorname{skel}^{n-3}\left(\text { Face }_{i} \sigma\right)} f^{\text {ids }(\sigma)}\left(\text { Face }_{i} \sigma\right) \sim k_{n-1}(n+1) \partial 0^{n}
$$

And since $z\left(\partial \sigma^{n}\right)=\partial 0^{n}$

$$
a\left(\partial \sigma^{n}\right) \sim\left(1+k_{n-1}(n+1)\right) \partial 0^{n}+\sum_{i=0}^{n}(-1)^{i} d^{\mathrm{ids}\left(\operatorname{Face}_{i} \sigma\right)}\left(\partial \operatorname{Face}_{i} \sigma\right)+\sum_{i=0}^{n}(-1)^{i} \sum_{\sigma \in \operatorname{skel}^{n-3}\left(\text { Face }_{i} \sigma\right)} f^{\mathrm{ids}(\sigma)}\left(\operatorname{Face}_{i} \sigma\right)
$$

Notice that if we prove

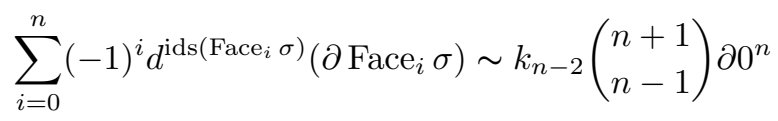

and

$$
\sum_{i=0}^{n}(-1)^{i} \sum_{\sigma \in \text { skel }^{n-3}\left(\text { Face }_{i} \sigma\right)} f^{\text {ids }(\sigma)}\left(\text { Face }_{i} \sigma\right) \sim \sum_{q=0}^{n-3} k_{q}\left(\begin{array}{c}
n+1 \\
q+1
\end{array}\right) \partial 0^{n}
$$

then

$$
a\left(\partial \sigma^{n}\right) \sim\left(1+\sum_{q=0}^{n-1} k_{q}\left(\begin{array}{c}
n+1 \\
q+1
\end{array}\right)\right) \partial 0^{n}
$$

Proof of equation (A.6). For $i, j \in[n]$ such that $i<j$, let $\alpha_{i j}$ be $(-1)^{i+j}\left(d^{\text {ids }\left(\text { Face }_{j} \sigma^{n}\right)}\left(\right.\right.$ Face $\left._{i j} \sigma^{n}\right)-d^{\text {ids( }\left(\text { Face }_{i} \sigma^{n}\right)}\left(\right.$ Face $\left.\left._{i j} \sigma^{n}\right)\right)$. The proof of Lemma 5.2 shows that $\sum_{i=0}^{n}(-1)^{i} d^{\text {ids }\left(\text { Face }_{i} \sigma^{n}\right)}\left(\partial\right.$ Face $\left._{i} \sigma^{n}\right)=\sum_{i=0}^{n} \sum_{j+1}^{n} \alpha_{i j}$, and also that $\alpha_{i j}$ is an $(n-1)$-cycle.

Consider $i, j \in[n]$ such that $i<j<n$. We have that

$$
\begin{aligned}
& \alpha_{i j}=(-1)^{i+j}\left(d^{\text {ids }\left(\text { Face }_{j} \sigma^{n}\right)}\left(\text { Face }_{i j} \sigma^{n}\right)-d^{\text {ids }\left(\text { Face }_{i} \sigma^{n}\right)}\left(\text { Face }_{i j} \sigma^{n}\right)\right) \\
& \alpha_{i j+1}=(-1)^{i+j+1}\left(d^{\text {ids }\left(\text { Face }_{j+1} \sigma^{n}\right)}\left(\text { Face }_{i j+1} \sigma^{n}\right)-d^{\text {ids }\left(\text { Face }_{i} \sigma^{n}\right.}\left(\text { Face }_{i j+1} \sigma^{n}\right)\right)
\end{aligned}
$$

It is easy to see that $\pi_{j}^{j+1}\left(\right.$ Face $\left._{i j} \sigma^{n}\right)=$ Face $_{i j+1} \sigma^{n}, \pi_{j}^{j+1}\left(\right.$ Face $\left._{j} \sigma^{n}\right)=$ Face $_{j+1} \sigma^{n}$ and $\pi_{j}^{j+1}\left(\right.$ Face $\left._{i} \sigma^{n}\right)=-$ Face $_{i} \sigma^{n}$. Thus, $\pi_{j}^{j+1}\left(\alpha_{i j}\right)=\alpha_{i j+1}$, because $d$ is equivariant. By Lemma A.2, for some integer $k_{i j}$,

$$
\alpha_{i j} \sim(-1)^{i} k_{i j} S_{i}
$$


It can be easily proved that $\pi_{j}^{j+1}\left(S_{i}\right)=-S_{i}$. Applying $\pi_{j}^{j+1}$ on both sides of Equation (A.8) and then multiplying by -1 , we get

$$
\alpha_{i j+1} \sim(-1)^{i} k_{i j+1} S_{i}
$$

By Lemma A.3 and Equations (A.8) and (A.9), $\alpha_{i j} \sim k_{i j} \partial 0^{n}$ and $\alpha_{i j+1} \sim k_{i j} \partial 0^{n}$. A similar analysis gives that, for every $i, j \in[n]$ such that $i<j-1, \alpha_{i j} \sim k_{i j} \partial 0^{n}$ and $\alpha_{i+1 j} \sim k_{i j} \partial 0^{n}$.

We can repeatedly use these two arguments to prove that $\alpha_{i j} \sim k_{i j} \partial 0^{n}$ and $\alpha_{i^{\prime} j^{\prime}} \sim k_{i j} \partial 0^{n}$, for every $i, i^{\prime}, j, j^{\prime} \in[n], i<j$ and $i^{\prime}<j^{\prime}$. Therefore, $\sum_{i=0}^{n} \sum_{j+1}^{n} \alpha_{i j} \sim\left(\begin{array}{c}n+1 \\ n-1\end{array}\right) k_{n-2} \partial 0^{n}$, for some integer $k_{n-2}$.

Proof of equation (A.7). The argument is very similar to the one used for Equation (A.6). The proof of Lemma 5.2 shows that

$$
\sum_{i=0}^{n}(-1)^{i} \sum_{\sigma \in \operatorname{skel}^{n-3}\left(\text { Face }_{i} \sigma\right)} f^{\operatorname{ids}(\sigma)}\left(\text { Face }_{i} \sigma\right)=\sum_{\sigma \in \operatorname{skel}^{n-3}\left(\sigma^{n}\right)} \sum_{i \in[n]-\operatorname{ids}(\sigma)}(-1)^{i} f^{\operatorname{ids}(\sigma)}\left(\text { Face }_{i} \sigma\right)
$$

Also it shows that $\sum_{i \in[n]-\operatorname{ids}(\sigma)}(-1)^{i} f^{\mathrm{ids}(\sigma)}\left(\mathrm{Face}_{i} \sigma\right)$ is an $(n-1)$-cycle. For each $\sigma \in \operatorname{skel}^{n-3}\left(\sigma^{n}\right)$, let $\alpha_{\sigma}$ be the cycle $\sum_{i \in[n]-\operatorname{ids}(\sigma)}(-1)^{i} f^{\mathrm{ids}(\sigma)}\left(\mathrm{Face}_{i} \sigma\right)$.

Consider $\sigma, \sigma^{\prime} \in \operatorname{skel}^{n-3}\left(\sigma^{n}\right)$ of same dimension, such that for some $P \subset[n]$ and $j \in[n], \operatorname{ids}(\sigma)=P \cup\{j\}, \operatorname{ids}\left(\sigma^{\prime}\right)=P \cup\{j+1\}$ and $j, j+1 \notin P$. Note

$$
\begin{aligned}
\alpha_{\sigma} & =\sum_{i \in[n]-\operatorname{ids}(\sigma)}(-1)^{i} f^{\mathrm{ids}(\sigma)}\left(\operatorname{Face}_{i} \sigma\right)=(-1)^{j+1} f^{\mathrm{ids}(\sigma)}\left(\text { Face }_{j+1} \sigma^{n}\right)+\sum_{i \in[n]-P}(-1)^{i} f^{\mathrm{ids}(\sigma)}\left(\text { Face }_{i} \sigma\right) \\
\alpha_{\sigma^{\prime}} & =\sum_{i \in[n]-\operatorname{ids}\left(\sigma^{\prime}\right)}(-1)^{i} f^{\mathrm{ids}\left(\sigma^{\prime}\right)}\left(\text { Face }_{i} \sigma\right)=(-1)^{j} f^{\mathrm{ids}\left(\sigma^{\prime}\right)}\left(\text { Face }_{j} \sigma\right)+\sum_{i \in[n]-P}(-1)^{i} f^{\mathrm{ids}\left(\sigma^{\prime}\right)}\left(\text { Face }_{i} \sigma\right)
\end{aligned}
$$

It is easy to see that $\pi_{j}^{j+1}(\sigma)=\sigma^{\prime}, \pi_{j}^{j+1}\left(\right.$ Face $\left._{j+1} \sigma^{n}\right)=$ Face $_{j} \sigma$ and $\pi_{j}^{j+1}\left(\right.$ Face $\left._{i} \sigma\right)=-$ Face $_{i} \sigma$ for each $i \in[n]-P$. Then, $\pi_{j}^{j+1}\left(\alpha_{\sigma}\right)=-\alpha_{\sigma^{\prime}}$, since $f$ is equivariant.

Fix an $i \in[n]-\operatorname{ids}(\sigma)$. By Lemma A.2, for some integer $k_{\sigma}$

$$
\alpha_{\sigma} \sim(-1)^{i} k_{\sigma} S_{i}
$$

It can be easily proved that $\pi_{j}^{j+1}\left(S_{i}\right)=-S_{i}$. Applying $\pi_{j}^{j+1}$ on both sides of Equation (A.11) and then multiplying by -1 , we get

$$
\alpha_{\sigma^{\prime}} \sim(-1)^{i} k_{\sigma} S_{i}
$$

By Lemma A.3 and Equations (A.10) and (A.11), $\alpha_{\sigma} \sim k_{\sigma} \partial 0^{n}$ and $\alpha_{\sigma^{\prime}} \sim k_{\sigma} \partial 0^{n}$. We can repeatedly use this argument to prove that $\alpha_{\sigma} \sim k_{\sigma} \partial 0^{n}$ and $\alpha_{\sigma^{\prime}} \sim k_{\sigma} \partial 0^{n}$, for every $\sigma, \sigma^{\prime} \in \operatorname{skel}^{n-3}\left(\sigma^{n}\right)$ of same dimension.

Therefore, $\sum_{\sigma \in \operatorname{skel}^{n-3}\left(\sigma^{n}\right)} \sum_{i \in[n]-\operatorname{ids}(\sigma)}(-1)^{i} f^{\operatorname{ids}(\sigma)}\left(\right.$ Face $\left._{i} \sigma\right) \sim \sum_{q=0}^{n-3} k_{q}\left(\begin{array}{l}n+1 \\ q+1\end{array}\right) \partial 0^{n}$.

\section{A.2 Proofs of Section 5.2}

Lemma A.4. Let $a: \mathcal{C}\left(\sigma^{n}\right) \rightarrow \mathcal{C}\left(\mathcal{A}^{n}\right)$ be the chain map induced by a chromatic and binary colored subdivision $\chi\left(\sigma^{n}\right)$ without monochromatic $n$-simplexes produced by the construction in [3]. Then, $a$ is non-trivial, color-preserving and equivariant.

Proof. Let $\mathcal{O}^{n}$ be the complex $\mathcal{A}^{n}$ with simplexes $\{(0,0), \ldots,(n, 0)\}$ and $\{(0,1), \ldots,(n, 1)\}$. Observe that $a$ is also a chain map $\mathcal{C}\left(\sigma^{n}\right) \rightarrow \mathcal{C}\left(\mathcal{O}^{n}\right)$. For technical reasons, we think of $a$ in this way.

First, since the subdivision $\chi\left(\sigma^{n}\right)$ is chromatic, clearly $a$ is non-trivial and color-preserving. By induction on $q$, we prove the following proposition.

Proposition A.5. The restriction $\left.a\right|_{\mathcal{C}\left(\operatorname{skel}^{q}\left(\sigma^{n}\right)\right)}, 0 \leq q \leq n$, is equivariant.

By symmetry of the binary coloring of $\chi\left(\sigma^{n}\right)$, Proposition A.5 clearly holds for $q=0$. Suppose that Proposition A.5 holds for dimension $q-1$. We prove it holds for dimension $q$.

By symmetry of the binary coloring of $\chi\left(\sigma^{n}\right)$, for the face $\sigma=\langle 0 \ldots q\rangle$ of $\sigma^{n}$ we have that $a \circ \pi_{i}^{q}\left(\right.$ Face $\left._{q} \sigma\right)=a\left(\right.$ Face $\left._{i} \sigma\right)=$ $\pi_{i}^{q} \circ a\left(\right.$ Face $\left._{q} \sigma\right)$. Therefore, if we prove that $\pi \circ a\left(\right.$ Face $\left._{q} \sigma\right)=a \circ \pi\left(\right.$ Face $\left._{q} \sigma\right)$ for every $\pi \in \mathcal{S}_{n}$, then $\pi \circ a\left(\right.$ Face $\left._{i} \sigma\right)=a \circ \pi\left(\right.$ Face $\left._{i} \sigma\right)$, since $a\left(\right.$ Face $\left._{i} \sigma\right)=\pi_{i}^{q} \circ a\left(\right.$ Face $\left._{q} \sigma\right)$.

Consider the face $\sigma=\langle 0 \ldots q\rangle$ of $\sigma^{n}$. Let $L_{q}$ be $\left\{\tau \mid \tau \in \operatorname{skel}^{q}\left(\mathcal{O}^{n}\right)\right.$ and $\left.\operatorname{ids}(\tau)=[q]\right\}$. For $\tau \in L_{q}$, let \#1( $\left.\#\right)$ be the number of its vertexes with binary color 1 , and let $\operatorname{inv}(\tau, i), 0 \leq i \leq q$, denote the simplex of $L_{q}$ with the same vertexes as $\tau$ but with the vertex with id $i$ having the opposite binary coloring to the binary coloring of the vertex with id $i$ of $\tau$. For $0 \leq k \leq q+1$, let $L_{q, k}$ denote the set $\left\{\tau \mid \tau \in L_{q}\right.$ and $\left.\# 1(\tau)=k\right\}$. Thus $\left|L_{q, k}\right|=\left(\begin{array}{c}q+1 \\ k\end{array}\right)$. Since $a$ is color-preserving, we can write $a(\sigma)=\sum_{\tau \in L_{q}} k_{\tau} \tau$, where $k_{\tau} \in \mathbb{Z}$. Obviously if $q=n$ then $k_{\{(0,0), \ldots,(n, 0)\}}=k_{\{(0,1), \ldots,(n, 1)\}}=0$, since $\mathcal{A}^{n}$ does not have monochromatic $n$-simplexes. We prove the following proposition. 
Proposition A.6. For every $\tau, \tau^{\prime} \in L_{q, k}, k_{\tau}=k_{\tau^{\prime}}, 0 \leq k \leq q+1$.

For example, for $\sigma=\langle 012\rangle$ and $k=2$, Proposition A.6 says that if $\langle(0,0)(1,1)(2,1)\rangle$ appears in $a(\sigma)$ with coefficient $\ell$, then $\langle(0,1)(1,1)(2,0)\rangle$ and $\langle(0,1)(1,0)(2,1)\rangle$ appear in $a(\sigma)$ with coefficient $\ell$ too. It is not hard to see that this proves $a \circ \pi(\sigma)=\pi \circ a(\sigma)$ for every $\pi \in \mathcal{S}_{n}$, hence Proposition A.5 holds for $q$.

We proceed by induction on $k$. For $k=0$ we have that $\left|L_{q, k}\right|=1$, thus Proposition A.6 trivially holds. Suppose Proposition A.6 holds for $k-1$. We prove it holds for $k$.

Notice that

$$
\partial a(\sigma)=\sum_{\tau \in L_{q}} k_{\tau} \partial \tau=\sum_{\tau \in L_{q}} k_{\tau} \sum_{i=0}^{q}(-1)^{i} \operatorname{Face}_{i} \tau=a(\partial \sigma)=\sum_{i=0}^{q}(-1)^{i} a\left(\text { Face }_{i} \sigma\right)
$$

where Face $_{i} \tau=\left\langle\left(0, b_{0}\right) \ldots \widehat{\left(i, b_{i}\right)} \ldots\left(q, b_{q}\right)\right\rangle$ for $\tau=\left\langle\left(0, b_{0}\right) \ldots\left(i, b_{i}\right) \ldots\left(q, b_{q}\right)\right\rangle$. Consider a simplex $\tau \in L_{q}$ and $i \in\{0, \ldots, q\}$. Observe that the $(q-1)$-simplex Face $_{i} \tau$ appears in $\partial a(\sigma)$ with coefficient $(-1)^{i}\left(k_{\tau}+k_{\operatorname{inv}(\tau, i)}\right)$, since Face $i \tau$ is face of $\tau$ and $\operatorname{inv}(\tau, i)$. Moreover, Face $_{i} \tau$ appears in $a\left(\right.$ Face $\left._{i} \sigma\right)$ with coefficient $k_{\tau}+k_{\operatorname{inv}(\tau, i)}$, because $\partial a(\sigma)=a(\partial \sigma)$ and $a$ is color-preserving. Also notice that either $\# 1(\tau)=\# 1\left(\right.$ Face $\left._{i} \tau\right)$ and $\# 1(\operatorname{inv}(\tau, i))=\# 1\left(\right.$ Face $\left._{i} \tau\right)+1$, or $\# 1(\tau)=\# 1\left(\right.$ Face $\left._{i} \tau\right)+1$ and $\# 1(\operatorname{inv}(\tau, i))=$ $\# 1\left(\right.$ Face $\left._{i} \tau\right)$.

Consider the set $N=\left\{\tau \mid \tau \in L_{q, k}\right.$ and $\# 1\left(\right.$ Face $\left.\left._{q} \tau\right)=k-1\right\}$. Note $|N|=\left(\begin{array}{c}q \\ k-1\end{array}\right)$. For each $\tau \in N$, observe that $\# 1(\operatorname{inv}(\tau, q))=$ $k-1$, hence $\operatorname{inv}(\tau, q) \in L_{q, k-1}$. Consider a simplex $\tau \in N$. As noticed above, Face $_{q} \tau$ appears in $a\left(\right.$ Face $\left._{q} \sigma\right)$ with coefficient $k_{\tau}+k_{\operatorname{inv}(\tau, q)}$. Consider $i \in\{0, \ldots, q\}$. Let $\rho_{i}$ and $\rho$ be the simplexes $\pi_{i}^{q}\left(\operatorname{Face}_{q} \tau\right)$ and $\pi_{i}^{q}(\tau)$. Observe that $\rho_{i}$ is a face of $\rho$, $\# 1\left(\rho_{i}\right)=k-1$ and $\# 1(\rho)=k$. As for Face $q$, we have that $\rho_{i}$ appears in $a\left(\right.$ Face $\left._{i} \sigma\right)$ with coefficient $k_{\rho}+k_{\operatorname{inv}(\rho, i)}$, where Face $_{i} \sigma=$ $\pi_{i}^{q}\left(\right.$ Face $\left._{q} \sigma\right)$. By the induction hypothesis, $\left.a\right|_{\mathcal{C}\left(\text { skel }^{q-1}\left(\sigma^{n}\right)\right)}$ is equivariant, hence $a \circ \pi_{i}^{q}\left(\right.$ Face $\left._{q} \sigma\right)=a\left(\right.$ Face $\left._{i} \sigma\right)=\pi_{i}^{q} \circ a\left(\right.$ Face $\left._{q} \sigma\right)$. Therefore, $k_{\tau}+k_{\operatorname{inv}(\tau, q)}=k_{\rho}+k_{\operatorname{inv}(\rho, i)}$. Moreover, $k_{\operatorname{inv}(\tau, q)}=k_{\operatorname{inv}(\rho, i)}$ because $\# 1(\operatorname{inv}(\tau, q))=\# 1(\operatorname{inv}(\rho, i))=k-1$ and, by the induction hypothesis, Proposition A.6 holds for $k-1$. Thus, we get $k_{\tau}=k_{\rho}$.

For each $\tau \in N$, let $M_{\tau}$ be $\left\{\pi_{i}^{q}(\tau) \mid 0 \leq i \leq q\right\}$. The previous paragraph proved that for every $\rho, \rho^{\prime} \in M_{\tau}, k_{\rho}=k_{\rho^{\prime}}$. It is not hard to see that $\left|M_{\tau}\right|=(q+1)-(k-1)$ for every $\tau \in N$, and $L_{q, k}=\cup_{\tau \in N} M_{\tau}$. Moreover, we have that the sets $M_{\tau}$ 's are not a partition of $L_{q, k}$ because $\frac{\left(\begin{array}{c}q+1 \\ k\end{array}\right)}{((q+1)-(k-1))\left(\begin{array}{c}q \\ k-1\end{array}\right)}=\frac{q+1}{k((q+1)-(k-1))}<1$. Thus, these sets intersect each other, hence $\tau, \tau^{\prime} \in L_{q, k}, k_{\tau}=k_{\tau^{\prime}}$. This completes the proof. 